\title{
Relevancia clínica de las interacciones medicamentosas en pacientes infectados con el virus de la inmunodeficiencia humana: actualización 2009-2014
}

\author{
Newar A. Giraldo, Pedro Amariles, Daniel E. Pino Marín y María José Faus
}

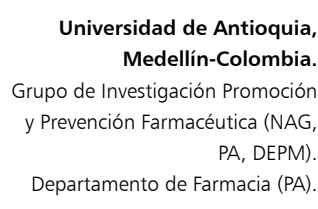

Universidad de Granada Granada-España Grupo de Investigación en Atención Farmacéutica (MJF)

Los autores declaran no tener conflictos de interés.

El Grupo de Investigación

Promoción y Prevención

Farmacéutica recibió apoyo

económico de la convocatoria de estrategia de sostenibilidad 2014-

2015, del Comité para el Desarrollo

de la Investigación (CODI) de la Universidad de Antioquia, Medellín-

Colombia.

Recibido: 30 de octubre de 2015 Aceptado: 23 de septiembre de

2016

Correspondencia a: Pedro Amariles pedro.amariles@udea.edu.co

\section{Introducción}

En pacientes con VIH/SIDA, la utilización de terapia anti-retroviral (TARV) combinada, debido a que incluye tres o más medicamentos anti-retrovirales (ARV), está asociada a la aparición de interacciones medicamentosas (IM), las que pueden generar problemas de seguridad o efectividad clínicamente relevantes, que pueden afectar en forma destacable los resultados alcanzados con la farmacoterapia en estos pacientes.

En el año $2007^{1}$ y en el $2010^{2}$ se publicaron revisiones, realizadas en la base de datos MEDLINE/PubMed hasta el 30 de abril de 2009 sobre IM de agentes ARV que han permitido identificar y valorar la relevancia clínica de este tipo de variable en la respuesta farmacológica. Adicionalmente, la información sistematizada en un capítulo de libro ha permitido establecer que, en el caso de las interacciones farmacocinéticas de relevancia clínica, $80 \%$ se explican por cambios en el aclaramiento sistémico (inhibición o inducción sistémica de la actividad metabólica de las isoformas CYP450) y $15 \%$ con cambios en la biodisponibilidad (variaciones en el $\mathrm{pH}$ gastrointestinal, en el metabolismo pre-sistémico por la CYP3A4 intestinal o hepática, o en la actividad de la glicoproteína-P) ${ }^{3}$.

Sin embargo, en los últimos años se han comercializado nuevos agentes ARV, cuya utilización terapéutica, debido a sus características farmacocinéticas, podría estar asociada a la presentación de IM relevantes clínicamente, previamente no identificadas o reportadas ${ }^{1-3}$. Por ello, se consideró adecuado actualizar dicha revisión hasta diciembre de 2014, utilizando la misma estrategia de búsqueda. En este sentido, el objetivo del presente trabajo fue actualizar la información previamente sistematizada sobre IM en pacientes con VIH/SIDA, verificando la funcionalidad de la propuesta para analizar y evaluar la relevancia clínica de las interacciones presentada anteriormente por los autores.

\section{Métodos}

Se realizó una revisión estructurada en MEDLINE/ PubMed de artículos publicados entre el 1 de mayo de 2009 y el 31 de diciembre de 2014 sobre IM de ARV en humanos. De manera similar, a las revisiones previas ${ }^{1,2}$, para la revisión se utilizaron los términos Mesh: Antiretroviral agents and drug interactions or herb-drug inte- 
ractions or food-drug interactions, en estudios realizados en humanos, publicados en inglés o español y con acceso a texto completo. La búsqueda fue complementada con la revisión e inclusión de las referencias de los artículos seleccionados considerados como relevantes.

La inclusión de los artículos fue evaluada por tres investigadores independientes $\mathrm{y}$, en caso de requerirlo, por consenso entre ellos.

La relevancia clínica de las interacciones medicamentosas se definió y evaluó utilizando la probabilidad de ocurrencia y la gravedad de la interacción ${ }^{1-4}$. La probabilidad de aparición de la interacción se estableció en tres categorías-, a partir del resultado, en la salud del paciente, de la interacción ${ }^{4}$ :

- Definida: Interacción documentada en meta-análisis, revisiones sistemáticas o ensayos clínicos de asignación aleatoria o no aleatoria.

- Probable: Interacción documentada en estudios analíticos o por la descripción de tres o más casos clínicos.

- Posible: Interacciones documentadas por la descripción de menos de tres casos o por recomendaciones de expertos.

Por su parte, la gravedad de la interacción se estableció en tres categorías, a partir del resultado en salud en el paciente de la interacción ${ }^{4}$ :

- Grave: La interacción puede causar daño o lesión al paciente. El resultado de la interacción puede causar o generar en el paciente la muerte, riesgo para la vida, hospitalización, una incapacidad permanente o significativa, anomalías congénitas o malformaciones al nacer.

- Moderada: La interacción genera la necesidad de realizar un seguimiento del paciente. El resultado de la interacción puede causar una modificación (cambio o interrupción) de la farmacoterapia o el empleo de nuevos fármacos para tratar el problema relacionado con medicamentos o bien la prolongación de la hospitalización.

- Leve: La interacción no causa daño al paciente. El resultado de la interacción no genera la necesidad de modificar (cambiar o suspender) la farmacoterapia, o el empleo de nuevos fármacos para tratar el problema relacionado con los medicamentos ni prolonga la hospitalización del paciente.

A partir de las combinaciones posibles de gravedad y probabilidad de aparición, las interacciones se agruparon en cuatro categorías:

- Nivel 1 (riesgo muy alto): Resultante de la combinación de grave y definida, o grave y probable. La utilización simultánea de los medicamentos se considera contraindicada "de forma absoluta".

- Nivel 2: (riesgo alto): Resultante de la combinación de grave y posible; moderada y definida o moderada y probable. La utilización simultánea de los medicamentos requiere el ajuste de la pauta posológica, valorar signos y síntomas de efectividad y seguridad del tratamiento, idealmente de forma cuantitativa.

- Nivel 3 (riesgo medio): Resultante de la combinación de moderada y posible; leve y definida, o leve y probable. La utilización simultánea de los medicamentos requiere el ajuste de la posología o valorar signos y síntomas de efectividad y seguridad del tratamiento, idealmente de forma cuantitativa.

- Nivel 4 (riesgo bajo): Resultante de la combinación leve y posible. La interacción es de escasa relevancia clínica.

\section{Resultados}

Con la estrategia de búsqueda se identificaron 546 artículos. Aunque, el acceso a texto completo hizo parte de los criterios de búsqueda, en el caso de 109 artículos identificados, el mismo no estaba disponible. Por ello, se accedió a 437 artículos completos, de los cuales 273 aportaban nuevas interacciones o actualizaciones a las revisiones previas realizadas ${ }^{1-3}$. Adicionalmente, se incluyeron 11 artículos identificados en las referencias de los artículos seleccionados, por tanto, para la presente revisión se utilizaron 284 artículos interacciones medicamentosas de ARV en humanos. En la Figura 1 se presenta el detalle del flujo de la revisión.

Se identificaron 935 parejas nuevas de IM, cuyas características se detallan en la Tabla 1. De este grupo, 861 (92\%) fueron parejas de interacciones medicamento/medicamento y 74 ( $8 \%$ ) de medicamentos con fitoterapéuticos, enfermedades, condiciones especiales (alteraciones renales, hepáticas y mujeres en estado de embarazo), alimentos o drogas de abuso. De las 935 parejas de interacciones, $895(95,7 \%)$ fueron farmacocinéticas, 561 (60\%) mediadas principalmente por la inhibición enzimática; mientras que de las 40 (4,3\%) farmacodinámicas, 32 se debieron a sinergismo. Con respecto al nivel de relevancia clínica, 402 interacciones $(43 \%)$ fueron valoradas con mayor riesgo de generar problemas de efectividad y seguridad de los medicamentos anti-retrovirales: $4(0,43 \%)$ de nivel 1 y $398(42.47 \%$ ) de nivel 2 (Tabla 1). Por su parte, el detalle de los pares de IM clínicamente relevantes (nivel 1 y 2) se presenta en las Tablas 2 a 6.

En la Tabla 2 se presentan las IM relacionadas con los inhibidores de proteasa (IP). En términos generales, estos ARV se comportan como inhibidores enzimáticos, aumentando las concentraciones plasmáticas de un número significativo de medicamentos, lo que hace que se deban monitorizar parámetros de seguridad de estos medicamentos. Por otra parte, el jugo de pomelo, doxiciclina y posaconazol son responsables de aumentar 
Figura 1. Esquema general del flujo y detalle del resultado de artículos identificados y seleccionados para la revisión.
Estrategia general de la búsqueda:

MEDLINE/PubMed (mayo de 2009 - diciembre de 2014) Términos Mesh: Anti-retroviral agents and drug interactions or herb-drug interactions or food-drug interactions

\section{Criterios de inclusión:}

Artículos con interacciones medicamentosas, en humanos, publicadas en español e inglés con acceso a texto completo

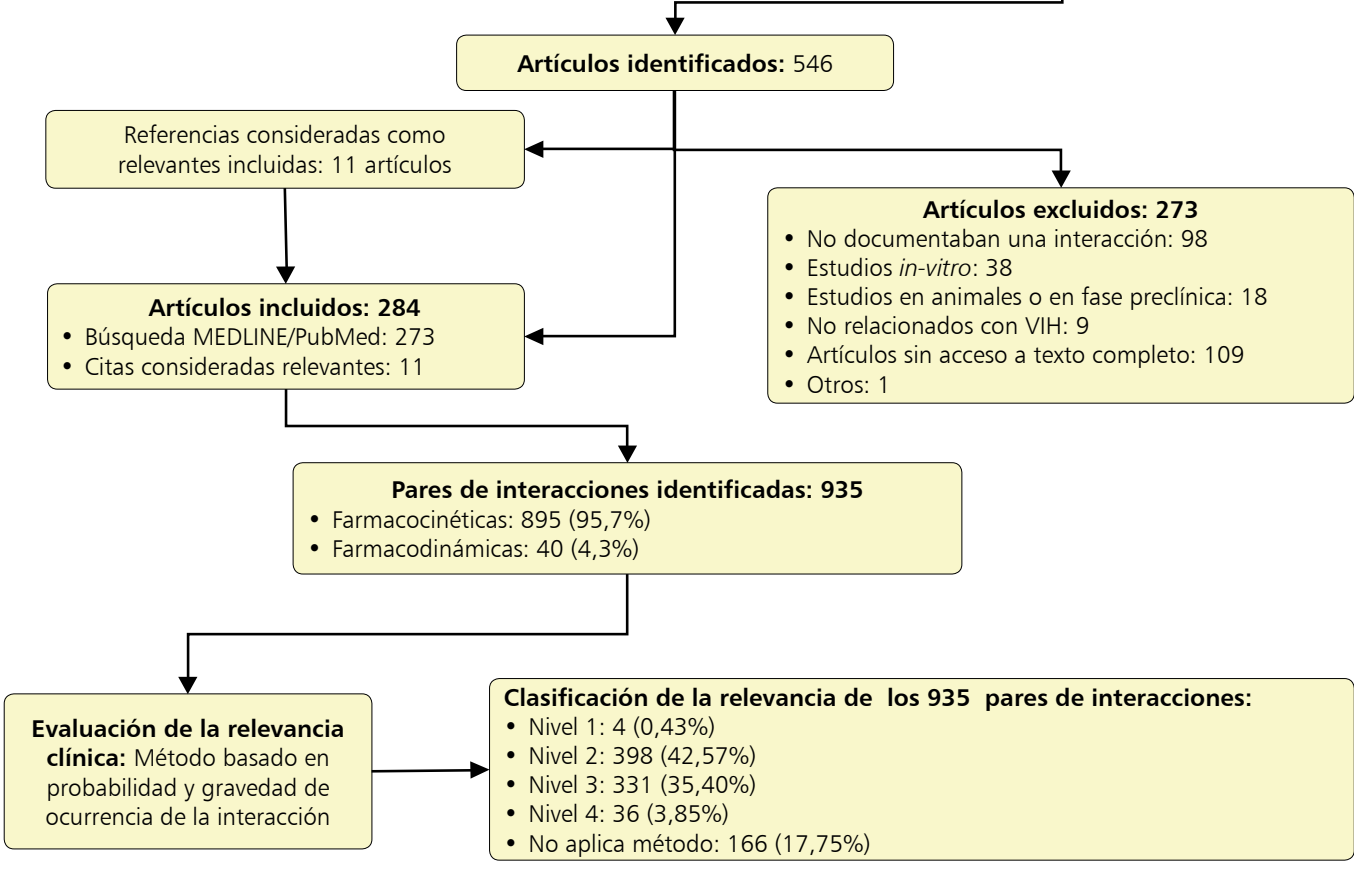

Tabla 1. Características de las interacciones medicamentosas en pacientes con VIH/SIDA

Cuadro resumen de las interacciones medicamentosas encontradas

\begin{tabular}{lrrr} 
Total parejas de interacciones & \multicolumn{3}{c}{$935(100,0)$} \\
\hline Mecanismo farmacocinético, n (\%) & 895 & $(95,7)$ \\
\hline Inhibición enzimática, n (\%) & 561 & $(60,0)$ \\
\hline Inducción enzimática, n (\%) & 262 & $(28,0)$ \\
\hline Cambio en biodisponibilidad, n (\%) & 67 & $(7,2)$ \\
\hline Inducción/inhibición enzimática, n (\%) & 5 & $(0,5)$ \\
\hline Mecanismo farmacodinámico, n (\%) & 40 & $(4,3)$ \\
Sinergismo,n (\%) & 32 & $(3,4)$ \\
\hline Antagonismo,n (\%) & 8 & $(0,9)$
\end{tabular}

Nivel de la relevancia clínica de la interacción medicamentosa

Nivel 2

Nivel 3

Nivel 4

No aplica

Total, n (\%)

$4(0,43)$
$398(42,57) \quad 331(34,40)$
$166(17,75)$

$935(100,0)$ 
Tabla 2. Interacciones medicamentosas de carácter inhibitorio relacionadas con inhibidores de proteasa

\begin{tabular}{|c|c|c|c|}
\hline $\begin{array}{l}\text { Grupo farmacológico o } \\
\text { medicamentos afectados }\end{array}$ & $\begin{array}{l}\text { Agente anti- } \\
\text { retroviral }\end{array}$ & $\begin{array}{l}\text { Relevancia } \\
\text { clínica: nivel }\end{array}$ & Comentarios - sugerencias \\
\hline $\begin{array}{l}\text { Alimentos } \\
\text { Jugo de pomelo }\end{array}$ & $\begin{array}{l}\text { Lopinavir/ritonavir } \\
\quad(\text { LPV/rtv) }\end{array}$ & 2: riesgo alto & $\begin{array}{l}\text { El jugo de pomelo puede aumentar las concentraciones plasmáticas de lopinavir/rtv - Monitorizar } \\
\text { parámetros de seguridad de lopinavir/rtv, un ajuste en la dosis puede ser necesario }\end{array}$ \\
\hline $\begin{array}{l}\text { Anestésicos } \\
\text { Propoxifeno }^{7} \\
\text { Tramadol }^{7}\end{array}$ & Ritonavir (RTV) & 2: riesgo alto & $\begin{array}{l}\text { Ritonavir puede aumentar las concentraciones plasmáticas de propoxifeno y tramadol - Monitorizar } \\
\text { parámetros de seguridad de propoxifeno y tramadol, un ajuste en la dosis puede ser necesario. }\end{array}$ \\
\hline $\begin{array}{l}\text { Anestésico/benzodiazepina } \\
\text { Midazolam } \\
6,8,9-13\end{array}$ & Darunavir (DRV) & 2: riesgo alto & $\begin{array}{l}\text { Darunavir puede aumentar las concentraciones plasmáticas de midazolam, aumentando la pro- } \\
\text { babilidad de depresión respiratoria, sedación y debilidad muscular - Monitorizar parámetros de } \\
\text { seguridad de midazolam. Oxazepam, lorazepam o temazepam son una alternativa, debido a que } \\
\text { se eliminan por conjugación con ácido glucurónico y se afectan escasamente por el uso simultáneo } \\
\text { de inhibidores de proteasa (IP) }\end{array}$ \\
\hline
\end{tabular}

\section{Ansiolíticos/benzodiazepinas}

Clonazepam ${ }^{14}$

Atazanavir (ATV)/ 2: riesgo alto

Fosamprenavir

(FPV)//LPV/rtV

Diazepam $6,8,11,14-16$

ATV/DRV/FPV/LPV/

2: riesgo alto

SQV de inhibidores de proteasa (IP)

\section{Ansiolítico/fenotiazinas}

Tioridazina ${ }^{6,17}$

ATV/FPV/LPV

2: riesgo alto
Los IP pueden aumentar las concentraciones plasmáticas del clonazepam - Monitorizar parámetros de seguridad de clonazepam, un ajuste en la dosis puede ser necesario

Los IP pueden aumentar las concentraciones plasmáticas de diazepam, aumentando el riesgo de hipotensión arterial y disminución de la conducción cardiaca - Monitorizar parámetros de seguridad de diazepam, un ajuste en la dosis puede ser necesario

\section{Anti-anginoso/anti-arrítmico}

Metoprolol $^{6}$
RTV 2: riesgo alto

Los IP pueden aumentar las concentraciones plasmáticas de tioridazina - Monitorizar parámetros de seguridad de tioridazina, un ajuste en la dosis puede ser necesario. Considerar como uso alternativo olanzapina arterial y bradicardia en los pacientes - Monitorizar la presión arterial, la frecuencia cardíaca y ajustar la dosis de metoprolol

\section{Anti-arrítimicos}

Amiodarona $6,10,11,16$

Dofetilida $^{17}$

ATV/FPV/LPV

ATV/FPV/LPV

2: riesgo alto

Dronedarona ${ }^{14}$

DRV

2: riesgo alto

Flecainida $a^{9,17}$

Lidocaína $6,7,9,11,14,1$

ATV/DRV/FPV/LPV/ 2: riesgo alto SQV/ RTV

Mexiletina $^{17}$

ATV/FPV/LPV

Procainamida $^{17}$

ATV/FPV/LPV

2: riesgo alto

2: riesgo alto

\section{Anti-arrítimicos}

Propafenona $6,9,14,17,18$

Quinidina $6,14,17,19$
ATV/DRV/FPV 2: riesgo alto
Darunavir puede aumentar las concentraciones plasmáticas de amiodarona, aumentando la probabilidad de problemas de seguridad, en especial gastrointestinales, musculares y en la conducción cardiaca - Monitorizar parámetros de seguridad de amiodarona, un ajuste en la dosis puede ser necesario

Los IP pueden aumentar las concentraciones plasmáticas de dofetilida, aumentando la probabilidad de problemas de seguridad, en especial gastrointestinales, musculares y en la conducción cardiaca - Monitorizar parámetros de seguridad de dofetilida, un ajuste en la dosis puede ser necesario Los IP pueden aumentar las concentraciones plasmáticas de dronedarona - Monitorizar parámetros de seguridad de dronedarona, un ajuste en la dosis puede ser necesario

Darunavir puede aumentar las concentraciones plasmática de flecainida aumentado la probabilidad de problemas de seguridad, en especial la conducción cardiaca - Monitorizar parámetros de seguridad de flecainida, un ajuste en la dosis puede ser necesario

Los IP pueden aumentar las concentraciones plasmáticas de lidocaína, aumentando la probabilidad de problemas de seguridad, en especial la conducción cardiaca - Monitorizar parámetros de seguridad de lidocaína, un ajuste en la dosis puede ser necesario

Los IP pueden aumentar las concentraciones plasmáticas de mexiletina - Monitorizar parámetros de seguridad de mexiletina, una disminución de la dosis puede ser necesario

Los IP pueden aumentar las concentraciones plasmáticas de procainamida y la probabilidad de problemas de seguridad, en especial la conducción cardiaca - Monitorizar parámetros de seguridad de procainamida, un ajuste en la dosis puede ser necesario

\section{Antiasmático/corticosteroides}

Budesonida ${ }^{14,20-23}$

Fluticasona $6,9,15,19,20,23-26$
ATV/FPV/LPV/rtV

ATV/DRV/FPV/

$\mathrm{LPV} / \mathrm{rtV}$
2: riesgo alto

2: riesgo alto

os IP pueden aumentar las concentraciones plasmáticas de propafenona y quinidina - Monitorizar parámetros de seguridad de propafenona y quinidina, un ajuste en la dosis puede ser necesario 


\begin{tabular}{|c|c|c|c|}
\hline \multicolumn{4}{|l|}{ Continuación Tabla 2} \\
\hline $\begin{array}{l}\text { Grupo farmacológico o } \\
\text { medicamentos afectados }\end{array}$ & $\begin{array}{l}\text { Agente anti- } \\
\text { retroviral }\end{array}$ & $\begin{array}{l}\text { Relevancia } \\
\text { clínica: nivel }\end{array}$ & Comentarios - sugerencias \\
\hline \multicolumn{4}{|c|}{ Antiasmático/corticosteroides } \\
\hline Triamcinolona $27-29$ & RTV & 2: riesgo alto & $\begin{array}{l}\text { Ritonavir puede aumentar las concentraciones plasmáticas de triamcinolona generando hipercorti- } \\
\text { solismo - Monitorizar parámetros de seguridad de triamcinolona, monitorizar los niveles de cortisol } \\
\text { y si es necesario suspender la triamcinolona }\end{array}$ \\
\hline \multicolumn{4}{|l|}{ Antimicrobiano/tetraciclinas } \\
\hline Doxiciclina 7,30 & ATV & 2: riesgo alto & $\begin{array}{l}\text { Doxiciclina puede aumentar las concentraciones plasmáticas de atazanavir - Monitorizar parámetros } \\
\text { de seguridad de este IP, un ajuste en la dosis puede ser necesario }\end{array}$ \\
\hline \multicolumn{4}{|l|}{ Anticoagulante oral } \\
\hline Rivaroxaban ${ }^{8}$ & LPV/rtv & 2: riesgo alto & $\begin{array}{l}\text { Lopinavir y ritonavir pueden aumentar las concentraciones plasmáticas de rivaroxaban generando } \\
\text { riesgo de hemorragias - Monitorizar parámetros de seguridad de rivaroxaban, un ajuste en la dosis } \\
\text { puede ser necesario }\end{array}$ \\
\hline Rivaroxaban ${ }^{31}$ & DRV & $\begin{array}{l}\text { 1: riesgo muy } \\
\quad \text { alto }\end{array}$ & $\begin{array}{l}\text { Darunavir puede aumentar las concentraciones plasmáticas de rivaroxaban y el riesgo de sangrado } \\
\text { gastrointestinal- monitorizar parámetros de seguridad de rivaroxaban, en especial sangrado gas- } \\
\text { trointestinal. Evitar en lo posible la combinación }\end{array}$ \\
\hline \multicolumn{4}{|l|}{ Anticonceptivo } \\
\hline Etonogestrel $^{32}$ & LPV/rtv & 2: riesgo alto & $\begin{array}{l}\text { Lopinavir/rtv puede aumentar las concentraciones plasmáticas de etonogestrel - Monitorizar pará- } \\
\text { metros de seguridad de etonogestrel, un ajuste en la dosis puede ser necesario. Además de utilizar } \\
\text { un método de anticoncepción alternativo }\end{array}$ \\
\hline \multicolumn{4}{|l|}{ Anticonvulsivantes } \\
\hline Carbamazepina9,11,15 & DRV & 2: riesgo alto & $\begin{array}{l}\text { Darunavir puede aumentar las concentraciones plasmáticas de carbamazepina - Monitorizar pará- } \\
\text { metros de seguridad de carbamazepina }\end{array}$ \\
\hline Mirtazapina ${ }^{13,17}$ & ATV/FPV & 2: riesgo alto & $\begin{array}{l}\text { Los IP pueden aumentar las concentraciones plasmáticas de mirtazapina - Monitorizar parámetros } \\
\text { de seguridad de mirtazapina, un ajuste en la dosis puede ser necesario }\end{array}$ \\
\hline Nefazodona ${ }^{17}$ & ATV/FPV & 2: riesgo alto & $\begin{array}{l}\text { Los IP pueden aumentar las concentraciones plasmáticas de nefazodona - Monitorizar parámetros } \\
\text { de seguridad de nefazodona, un ajuste en la dosis puede ser necesario }\end{array}$ \\
\hline Trazodona $6,13,14,17,33$ & FPV & 2: riesgo alto & $\begin{array}{l}\text { Los IP pueden inhibir el metabolismo de trazodona, lo que puede llevar a la aparición de problemas } \\
\text { de toxicidad, especialmente disminución de la conducción y paro cardiaco, como el aumento de } \\
\text { efectos anticolinérgicos (estreñimiento, boca seca, retención urinaria) - Monitorizar parámetros de } \\
\text { seguridad de trazodona, un ajuste en la dosis puede ser necesario }\end{array}$ \\
\hline \multicolumn{4}{|l|}{ Anticonvulsivante } \\
\hline Vilazodona $^{17}$ & ATV/FPV/ LPV & 2: riesgo alto & $\begin{array}{l}\text { Los IP pueden aumentar las concentraciones plasmáticas de vilazodona - Monitorizar parámetros de } \\
\text { seguridad de vilazodona, se puede disminuir la dosis a } 20 \mathrm{mg} \text { una vez al día }\end{array}$ \\
\hline \multicolumn{4}{|l|}{ Antidepresivos tricíclicos } \\
\hline $\begin{array}{l}\text { Amitriptilina } \\
\text { Desipramina }^{13} \\
\text { Nortriptilina }^{13}\end{array}$ & ATV/FPV & 2: riesgo alto & $\begin{array}{l}\text { Estos IP pueden aumentar las concentraciones plasmáticas de amitriptilina, desipramina y nortrip- } \\
\text { tilina - Monitorizar parámetros de seguridad de amitriptilina, desipramina y nortriptilina, un ajuste } \\
\text { en la dosis puede ser necesario }\end{array}$ \\
\hline Clomipramina ${ }^{13}$ & ATV/FPV/ & 2: riesgo alto & Los IP pueden aumentar las concentraciones plasmáticas de clomipramina, doxepina e imipramina \\
\hline $\begin{array}{l}\text { Doxepina }{ }^{13} \\
\text { Imipramina }\end{array}$ & LPV/rtv & & $\begin{array}{l}\text { - Monitorizar parámetros de seguridad de clomipramina, doxepina e imipramina. Un ajuste en la } \\
\text { dosis puede ser necesario }\end{array}$ \\
\hline \multicolumn{4}{|c|}{$\begin{array}{l}\text { Antidepresivos/inhibidores de la recaptación } \\
\text { de noradrenalina/serotonina }\end{array}$} \\
\hline Desvenlafaxina ${ }^{17,34}$ & ATV/FPV/LPV & 2: riesgo alto & $\begin{array}{l}\text { Los IP pueden aumentar las concentraciones plasmáticas de desvenlafaxina - Monitorizar parámetros } \\
\text { de seguridad de desvenlafaxina, un ajuste en la dosis podría ser necesario }\end{array}$ \\
\hline Duloxetina $^{13,17}$ & $\begin{array}{l}\text { ATV/DRV/FPV/ } \\
\text { LPV/rtV }\end{array}$ & 2: riesgo alto & $\begin{array}{l}\text { Los IP pueden aumentar las concentraciones plasmáticas de duloxetina- Monitorizar parámetros de } \\
\text { seguridad de duloxetina, un ajuste en la dosis puede ser necesario }\end{array}$ \\
\hline Fluvoxamina $^{13}$ & FPV & 2: riesgo alto & $\begin{array}{l}\text { Este IP pueden inhibir la CYP2D6 y, con ello, el metabolismo de fluvoxamina, lo que puede llevar a } \\
\text { la aparición de problemas de toxicidad de fluvoxamina - Monitorizar parámetros de seguridad de } \\
\text { fluvoxamina, una disminución en la dosis puede ser necesario }\end{array}$ \\
\hline Paroxetina $^{13}$ & ATV & 2: riesgo alto & $\begin{array}{l}\text { Este IP pueden aumentar las concentraciones plasmáticas de paroxetina - Monitorizar parámetros } \\
\text { de seguridad de paroxetina, un ajuste en la dosis puede ser necesario }\end{array}$ \\
\hline \multicolumn{4}{|l|}{ Antidiabéticos/gliptinas } \\
\hline Linagliptina ${ }^{17}$ & ATV/FPV/LPV & 2: riesgo alto & $\begin{array}{l}\text { Los IP pueden aumentar las concentraciones plasmáticas de linagliptina - Monitorizar parámetros } \\
\text { de seguridad de linagliptina, considerar el uso de sitagliptina }\end{array}$ \\
\hline Saxagliptina ${ }^{17}$ & ATV/FPV/LPV & 2: riesgo alto & $\begin{array}{l}\text { Los IP pueden aumentar las concentraciones plasmáticas de saxagliptina - Monitorizar parámetros } \\
\text { de seguridad de saxagliptina, un ajuste en la dosis puede ser necesario. La dosis de saxagliptina no } \\
\text { debe superar los } 2,5 \mathrm{mg} / \text { día }\end{array}$ \\
\hline
\end{tabular}




\begin{tabular}{|c|c|c|c|}
\hline \multicolumn{4}{|l|}{ Continuación Tabla 2} \\
\hline $\begin{array}{l}\text { Grupo farmacológico o } \\
\text { medicamentos afectados }\end{array}$ & $\begin{array}{l}\text { Agente anti- } \\
\text { retroviral }\end{array}$ & $\begin{array}{l}\text { Relevancia } \\
\text { clínica: nivel }\end{array}$ & Comentarios - sugerencias \\
\hline $\begin{array}{l}\text { Antidiabéticos/meglitinida } \\
\text { Nateglinida }^{17} \\
\text { Repaglinida }^{17}\end{array}$ & ATV/FPV /LPV & 2: riesgo alto & $\begin{array}{l}\text { Los IP pueden aumentar las concentraciones plasmáticas de nateglinida y repaglinida - Monitorizar } \\
\text { parámetros de seguridad de nateglinida y repaglinida, un ajuste en la dosis puede ser necesario }\end{array}$ \\
\hline \multicolumn{4}{|c|}{ Anti-diabético/tiazolidinedionas } \\
\hline Pioglitazona ${ }^{17}$ & ATV/FPV/LPV & 2: riesgo alto & $\begin{array}{l}\text { Los IP pueden aumentar las concentraciones plasmáticas de pioglitazona - Monitorizar parámetros } \\
\text { de seguridad de pioglitazona, un ajuste en la dosis puede ser necesario. Considerar el uso de rosi- } \\
\text { glitazona }\end{array}$ \\
\hline
\end{tabular}

Anti-hipertensivo/ bloqueador

de canales de calcio

Bepridil14,17 ATV/FPV/LPV 2: riesgo alto

Lacidipina $^{14} \quad$ RTV 2: riesgo alto

Los IP pueden aumentar las concentraciones plasmáticas de bepridil - Monitorizar parámetros de seguridad de bepridil, un ajuste en la dosis puede ser necesario

Ritonavir puede aumentar las concentraciones plasmáticas de lacidipina generando hipotensión arterial y bradicardia en los pacientes - Monitorizar la presión arterial, la frecuencia cardiaca y ajustar la dosis de lacidipina

\section{Anti-histamínico/anti-H1}

Astemizol ${ }^{6}$
DRV 1: riesgo muy alto

Darunavir puede aumentar las concentraciones de astemizol, aumentando la probabilidad de aumento del intervalo QTc en el electrocardiograma y aparición de arritmias cardiacas, al igual que alteraciones gastrointestinales y mareo- Monitorizar parámetros de seguridad de astemizol, evitar en lo posible la combinación

\section{Anti-infeccioso/anti-parasitario \\ Metronidazol $^{7}$}

\section{Antiinflamatorios esteroideos}

Prednisolona $7,20,28$

Anti-leproso

Artesunato ${ }^{11,18}$

Lumefantrina $9,11,35-38$
ATV/FPV/ 2: riesgo alto

LPV/RTV alto

1: riesgo muy Ritonavir puede aumentar las concentraciones plasmáticas de prednisolona - Monitorizar parámetros

Los IP pueden aumentar las concentraciones plasmáticas de metronidazol - Monitorizar parámetros de seguridad de metronidazol, un ajuste en la dosis puede ser necesario de seguridad de prednisolona, evitar en lo posible la combinación

DRV/LPV 2: riesgo alto

\section{Antimaláricos/aminoquinolona \\ Cloroquina $^{39}$}

LPV/RTV 2: riesgo alto

Ritonavir puede aumentar el área bajo la curva de artesunato en 28\% - Monitorizar parámetros de seguridad de artesunato, un ajuste en la dosis puede ser necesario

Ambos IP pueden aumentar las concentraciones plasmáticas de lumefantrina - Monitorizar parámetros de seguridad de lumefantrina, en especial el aumento del intervalo QT. Un ajuste en la dosis puede ser necesario

\section{Antimicótico}

Posaconazol ${ }^{40,41}$

ATV/RTV 2: riesgo alto

Lopinavir/rtv puede aumentar las concentraciones plasmáticas de cloroquina - Monitorizar parámetros de seguridad de cloroquina, un ajuste en la dosis puede ser necesario

\section{Anti-migrañosos/alcaloides}

\section{del ergot}

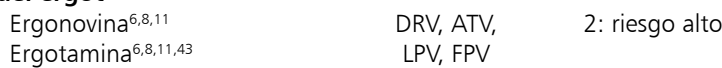

Posaconazol puede aumentar las concentraciones plasmáticas de atazanavir y ritonavir, mientras que ritonavir puede aumentar los niveles plasmáticos de posaconazol. Monitorizar parámetros de seguridad de atazanavir o ritonavir, y de posaconazol, un ajuste en la dosis puede ser necesario

Los IP pueden aumentar las concentraciones plasmáticas de ergonovina y ergotamina, aumentando la probabilidad de ergotismo: hipertensión arterial, nerviosismo, alucinaciones, convulsiones, alteraciones gastrointestinales y musculares - Su uso simultaneo está contraindicado

\section{Anti-neoplásicos \\ Irinotecan ${ }^{6,11,19}$}

Vincristina ${ }^{43}$
ATV

LPV/rtv
2: riesgo alto

2: riesgo alto

Atazanavir puede aumentar las concentraciones de irinotecan - Monitorizar parámetros de seguridad de irinotecan, un ajuste en la dosis puede ser necesario

Lopinavir/rtv puede aumentar la concentración máxima de vincristina - Monitorizar parámetros de seguridad de vincristina, una disminución de la dosis puede ser necesaria

\section{Anti-psicóticos/atípicos}

Risperidona ${ }^{13-17}$

Ziprasidona ${ }^{6,17,33}$
ATV/FPV/LPV

2: riesgo alto

LPV/rtv os IP pueden aumentar las concentraciones plasmáticas de risperidona - Monitorizar parámetros de seguridad de risperidona, un ajuste en la dosis puede ser necesario. Considerar el uso de olanzapina. Estos IP pueden aumentar las concentraciones plasmáticas de ziprasidona - Monitorizar parámetros de seguridad de ziprasidona, un ajuste en la dosis puede ser necesario 


\begin{tabular}{|c|c|c|c|}
\hline \multicolumn{4}{|l|}{ Continuación Tabla 2} \\
\hline $\begin{array}{l}\text { Grupo farmacológico o } \\
\text { medicamentos afectados }\end{array}$ & $\begin{array}{l}\text { Agente anti- } \\
\text { retroviral }\end{array}$ & $\begin{array}{l}\text { Relevancia } \\
\text { clínica: nivel }\end{array}$ & Comentarios - sugerencias \\
\hline \multicolumn{4}{|l|}{ Anti-psicóticos/típicos } \\
\hline Clorpromazina ${ }^{13,17}$ & $\begin{array}{l}\text { ATV/FPV/ } \\
\text { LPV/rtV }\end{array}$ & 2: riesgo alto & $\begin{array}{l}\text { Los IP pueden aumentar las concentraciones plasmáticas de clorpromazina - Monitorizar parámetros } \\
\text { de seguridad de clorpromazina, se puede considerar el uso de olanzapina }\end{array}$ \\
\hline Flufenazina ${ }^{17}$ & ATV/FPV/LPV & 2: riesgo alto & $\begin{array}{l}\text { Los IP pueden aumentar las concentraciones plasmáticas de flufenazina- Monitorizar parámetros } \\
\text { de seguridad de flufenazina, un ajuste en la dosis puede ser necesario. Considerar el uso alternativo } \\
\text { de olanzapina }\end{array}$ \\
\hline $\begin{array}{l}\text { Haloperido|6,13,17 } \\
\text { Perfenazina }{ }^{17}\end{array}$ & ATV/FPV/LPV & 2: riesgo alto & $\begin{array}{l}\text { Los IP pueden aumentar las concentraciones plasmáticas de haloperidol y perfenazina - Monitorizar } \\
\text { parámetros de seguridad de haloperidol y perfenazina, un ajuste en la dosis puede ser necesario }\end{array}$ \\
\hline |loperidona ${ }^{13,17,33}$ & $\begin{array}{l}\text { ATV/FPV/ } \\
\text { LPV/rtV }\end{array}$ & 2: riesgo alto & $\begin{array}{l}\text { Los IP pueden aumentar las concentraciones plasmáticas de iloperidona, aumentando la posibilidad } \\
\text { de prolongación del intervalo QT- Monitorizar parámetros de seguridad de iloperidona, un ajuste } \\
\text { en la dosis puede ser necesario }\end{array}$ \\
\hline Pimozida ${ }^{6}$ & DRV & 2: riesgo alto & $\begin{array}{l}\text { Darunavir puede aumentar las concentraciones plasmáticas de pimozida, generando mayor } \\
\text { probabilidad de aparición de movimientos involuntarios, agitación, confusión, alteraciones en el } \\
\text { comportamiento y taquicardia - Monitorizar parámetros de seguridad de pimozida, un ajuste en la } \\
\text { dosis puede ser necesario }\end{array}$ \\
\hline \multicolumn{4}{|l|}{ Hipnótico/benzodiazepinas } \\
\hline Flurazepam $6,11,14$ & $\begin{array}{l}\text { ATV/FPV/ } \\
\text { LPV/rtV }\end{array}$ & 2: riesgo alto & $\begin{array}{l}\text { Los IP pueden aumentar las concentraciones plasmáticas de flurazepam - Monitorizar parámetros } \\
\text { de seguridad de flurazepam, un ajuste en la dosis puede ser necesario }\end{array}$ \\
\hline \multicolumn{4}{|l|}{ Hipnótico/benzodiazepinas } \\
\hline Triazolam ${ }^{6,8-11,16}$ & DRV & 2: riesgo alto & $\begin{array}{l}\text { Darunavir puede aumentar las concentraciones plasmáticas de triazolam, aumentando la probabili- } \\
\text { dad de depresión respiratoria, sedación y debilidad muscular- Monitorizar parámetros de seguridad } \\
\text { de triazolam. Oxazepam, lorazepam o temazepam son una alternativa, debido a que se eliminan por } \\
\text { conjugación con ácido glucurónico y se afectan escasamente por el uso simultáneo de IP }\end{array}$ \\
\hline Zaleplon ${ }^{13}$ & $\begin{array}{l}\text { ATV/FPV/ } \\
\text { LPV/rtV }\end{array}$ & 2: riesgo alto & $\begin{array}{l}\text { Los IP pueden aumentar las concentraciones plasmáticas de zaleplon - Monitorizar parámetros de } \\
\text { seguridad de zaleplon, un ajuste en la dosis puede ser necesario }\end{array}$ \\
\hline \multicolumn{4}{|l|}{ Hipolipemiante/estatina } \\
\hline Lovastatina ${ }^{6,8,9,47}$ & $\begin{array}{l}\text { DRV, ATV, } \\
\text { LPV, FPV }\end{array}$ & 2: riesgo alto & $\begin{array}{l}\text { Los IP pueden aumentar las concentraciones plasmáticas de lovastatina, aumentando el riesgo de } \\
\text { miopatías y rabdomiólisis - No se recomienda su co-administración }\end{array}$ \\
\hline Simvastatina $a^{6,8,9,47}$ & $\begin{array}{l}\text { DRV, ATV, } \\
\text { LPV, FPV }\end{array}$ & 2: riesgo alto & $\begin{array}{l}\text { Los IP pueden aumentar las concentraciones plasmáticas de simvastatina, aumentando el riesgo de } \\
\text { miopatías y rabdomiólisis - No se recomienda su co-administración }\end{array}$ \\
\hline \multicolumn{4}{|l|}{$\begin{array}{l}\text { Inductor de sueño/hipnóticos } \\
\text { no barbitúricos }\end{array}$} \\
\hline Eszopiclona ${ }^{13}$ & ATV/FPV/ & 2: riesgo alto & Los IP pueden aumentar las concentraciones plasmáticas de eszopiclona, zolpidem y zopiclona - \\
\hline $\begin{array}{l}\text { Zolpidem }{ }^{6,13} \\
\text { Zopiclona }^{13}\end{array}$ & LPV/rtv & & $\begin{array}{l}\text { Monitorizar parámetros de seguridad de estos inductores de sueño, un ajuste en la dosis puede } \\
\text { ser necesario }\end{array}$ \\
\hline \multicolumn{4}{|l|}{ Procinético } \\
\hline Cisaprida $^{6}$ & DRV & 2: riesgo alto & $\begin{array}{l}\text { Darunavir puede aumentar las concentraciones de cisaprida, aumentando la probabilidad de aumen- } \\
\text { to del intervalo QTc y la aparición de arritmias cardiacas, al igual que alteraciones gastrointestinales } \\
\text { y mareo - Monitorizar parámetros de seguridad de cisaprida, un ajuste en la dosis del medicamento } \\
\text { puede ser necesario }\end{array}$ \\
\hline \multicolumn{4}{|c|}{ Psico-estimulante/drogas de abuso } \\
\hline $\begin{array}{l}3,4-\text { metilendioximetanfetami- } \\
\text { na (MDMA) }{ }^{11,49-50}\end{array}$ & RTV & 2: riesgo alto & $\begin{array}{l}\text { Ritonavir puede aumentar las concentraciones plasmáticas y la exposición a MDMA - Monitorizar } \\
\text { parámetros de seguridad de MDMA }\end{array}$ \\
\hline \multicolumn{4}{|l|}{$\begin{array}{l}\text { Tratamiento de la hiperplasia } \\
\text { prostática }\end{array}$} \\
\hline Alfuzosina $6,14,17,19$ & $\begin{array}{l}\text { ATV/DRV/ } \\
\text { FPV/LPV/rtV }\end{array}$ & 2: riesgo alto & $\begin{array}{l}\text { Los IP pueden aumentar las concentraciones plasmáticas de alfuzosina- Monitorizar parámetros de } \\
\text { seguridad de alfuzosina, un ajuste en la dosis puede ser necesario }\end{array}$ \\
\hline \multicolumn{4}{|l|}{$\begin{array}{l}\text { Tratamiento de la hiperplasia } \\
\text { prostática }\end{array}$} \\
\hline Tamsulosina ${ }^{14,17}$ & $\begin{array}{l}\text { ATV/FPV/ } \\
\text { LPV/rtV }\end{array}$ & 2: riesgo alto & $\begin{array}{l}\text { Los IP pueden aumentar las concentraciones plasmáticas de tamsulosina - Monitorizar parámetros } \\
\text { de seguridad de tamsulosina, un ajuste en la dosis puede ser necesario }\end{array}$ \\
\hline \multicolumn{4}{|c|}{$\begin{array}{l}\text { Los inhibidores de proteasa (ritonavir }>\text { atazanavir }>\text { fosamprenavir }>\text { darunavir), por inhibición fundamentalmente de citocromo CYP3A4, pueden disminuir el metabo- } \\
\text { lismo de otros fármacos, lo que puede causar un aumento en sus concentraciones plasmáticas y efectos tóxicos, DRV (darunavir), FPV (fosamprenavir), LPV (lopinavir), RTV } \\
\text { (ritonavir), ATV (atazanavir), MDMA (3,4-metilendioximetanfetamina). }\end{array}$} \\
\hline
\end{tabular}


Tabla 3. Interacciones medicamentosas de carácter inductor relacionadas con inhibidores de proteasa

\begin{tabular}{|c|c|c|c|}
\hline $\begin{array}{l}\text { Grupo farmacológico o } \\
\text { medicamentos afectados }\end{array}$ & $\begin{array}{l}\text { Agente anti- } \\
\text { retroviral }\end{array}$ & $\begin{array}{l}\text { Relevancia } \\
\text { clínica: nivel }\end{array}$ & Comentarios - sugerencias \\
\hline $\begin{array}{l}\text { Analgésico opioide } \\
\text { Metadona } 11,13,14,49,51-55\end{array}$ & LPV & 2 : riesgo alto & $\begin{array}{l}\text { Lopinavir/rtv puede disminuir el área bajo la curva de metadona en } 36,40 \text { y } 50 \% \text { respectivamente. } \\
\text { Monitorizar parámetros de efectividad de metadona, un ajuste en la dosis puede ser necesario }\end{array}$ \\
\hline $\begin{array}{l}\text { Ansiolíticos/benzodiazepinas } \\
\text { Lorazepam }^{13} \\
\text { Oxazepam }\end{array}$ & RTV & 2: riesgo alto & $\begin{array}{l}\text { Ritonavir puede disminuir las concentraciones plasmáticas de lorazepam y oxazepam, por inducción } \\
\text { de la glucoronidación - Monitorizar parámetros de efectividad de lorazepam y oxazepam, un ajuste } \\
\text { en la dosis puede ser necesario }\end{array}$ \\
\hline $\begin{array}{l}\text { Anti-coagulante oral } \\
\text { Eltrombopag }\end{array}$ & $\begin{array}{l}\text { Lopinavir/ritonavir } \\
\quad(\mathrm{LPV} / \mathrm{rtv})\end{array}$ & 2: riesgo alto & $\begin{array}{l}\text { Lopinavir/rtv puede disminuir el área bajo la curva de eltrombopag en } 17 \% \text { - Monitorizar paráme- } \\
\text { tros de efectividad de eltrombopag, a través del conteo de plaquetas y ajustar la dosis puede ser } \\
\text { necesario }\end{array}$ \\
\hline $\begin{array}{l}\text { Anti-conceptivos } \\
\text { Etinilestradiol6,9,11,57- } 63\end{array}$ & DRV/FPV/LPV/ & 2: riesgo alto & $\begin{array}{l}\text { Estos IP pueden disminuir las concentraciones plasmáticas de etinilestradiol - Monitorizar parámetros } \\
\text { de efectividad de etinilestradiol. Se debe recomendar a la paciente emplear un método barrera como } \\
\text { método complementario de planificación }\end{array}$ \\
\hline Noretindrona ${ }^{9,11,59,60,63}$ & LPV/rtv & 2: riesgo alto & $\begin{array}{l}\text { Darunavir puede disminuir las concentraciones plasmáticas de noretindrona y el efecto anticoncep- } \\
\text { tivo se puede ver disminuido- Monitorizar parámetros de efectividad de noretindrona. Se debe re- } \\
\text { comendar a la paciente emplear un método barrera como método complementario de planificación } \\
\text { En pacientes que utilizan IP, se recomienda anticonceptivos sólo con progestágeno } \\
\text { Lopinavir/rtv puede disminuir las concentraciones plasmáticas de norgestimato y el efecto anti- } \\
\text { conceptivo se puede ver disminuido - Monitorizar parámetros de efectividad de norgestimato. } \\
\text { Se debe recomendar a la paciente emplear un método barrera como método complementario de } \\
\text { planificación }\end{array}$ \\
\hline $\begin{array}{l}\text { Anticonvulsivante } \\
\text { Fenitoína } 6,10,11,15,64\end{array}$ & DRV & 2 : riesgo alto & $\begin{array}{l}\text { Fenitoína puede inducir la CYP3A4, aumentar el metabolismo de darunavir y disminuir sus concen- } \\
\text { traciones plasmáticas - Monitorizar parámetros de efectividad de darunavir, se recomienda utilizar } \\
\text { anticonvulsivantes de segunda generación (gabapentina, lamotrigina, vigabatrina y topiramato). } \\
\text { Gabapentina, al no ser metabolizada por la CYP, es considerada la más adecuada }\end{array}$ \\
\hline Fenobarbital $^{6}$ & DRV & 2: riesgo alto & $\begin{array}{l}\text { Fenobarbital puede inducir la CYP3A4 y puede disminuir las concentraciones plasmáticas de daru- } \\
\text { navir - Monitorizar parámetros de efectividad de darunavir, se recomienda utilizar anticonvulsivantes } \\
\text { de segunda generación (gabapentina, lamotrigina, vigabatrina y topiramato). Gabapentina,por no }\end{array}$ \\
\hline
\end{tabular}

\section{Anti-depresivos/inhibidores de la recaptación}

de noradrenalina-serotonina

Duloxetina ${ }^{13,17}$

LPV $\quad$ 2: riesgo alto

Paroxetina ${ }^{9,13,14,17,33,58,60,63}$

DRV/FPV

2: riesgo alto

Lopinavir/r puede disminuir las concentraciones plasmáticas de duloxetina - Monitorizar parámetros de efectividad de duloxetina, un ajuste en la dosis puede ser necesario

Ambos IP pueden disminuir las concentraciones plasmáticas de paroxetina - Monitorizar parámetros de efectividad de paroxetina y aumentar la dosis si es necesario

Anti-inflamatorio esteroideo

Dexametasona ${ }^{6,15}$

ATV/FPV /LPV/ 2: riesgo alto RTV/

Dexametasona puede disminuir las concentraciones plasmáticas de los IP - Monitorizar parámetros de efectividad de los IP, un ajuste en la dosis puede ser necesario

Anti-maláricos
Artemeter $35,36,38,65$
LPV/rtv $\quad$ 2: riesgo alto

Lopinavir/r puede disminuir las concentraciones plasmáticas de artemeter - Monitorizar parámetros de efectividad de artemeter, un ajuste en la dosis puede ser necesario

Anti-micótico

Posaconazol ${ }^{41,66,67}$ FPV 2: riesgo alto

Fosamprenavir disminuye las concentraciones plasmáticas de posaconazol - Monitorizar parámetros de efectividad de posaconazol. No se recomienda dar estos dos medicamentos juntos, sin presencia de ritonavir

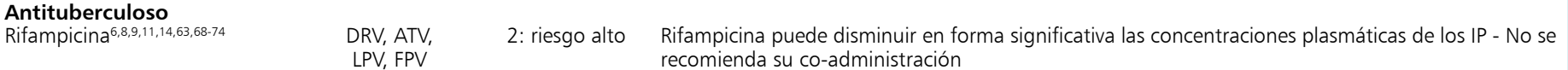

Hipnótico/benzodiazepinas

Temazepam ${ }^{13}$

RTV 2: riesgo alto

recomienda su co-administración

$\begin{array}{ll} & \text { coronidación - } \\ & \text { ser necesario }\end{array}$

\section{Hipolipemiante/fibrato}

Gemfibrozilo ${ }^{47,75} \quad$ LPV/rtv $\quad$ 2: riesgo alto

opinavir/rtv puede disminuir las concentraciones plasmáticas de gemfibrozilo - Monitorizar parámetros de efectividad de gemfibrozilo, un ajuste en la dosis puede ser necesario

Hierbas
Hierba de San Juan ${ }^{6,8,9} \quad$ DRV, ATV, 1 : riesgo muy

La hierba de San Juan puede inducir la CYP3A4 y aumentar el metabolismo de los IP, lo que puede causar una disminución en sus concentraciones plasmáticas y en la respuesta virológica - No se recomienda su co-administración 


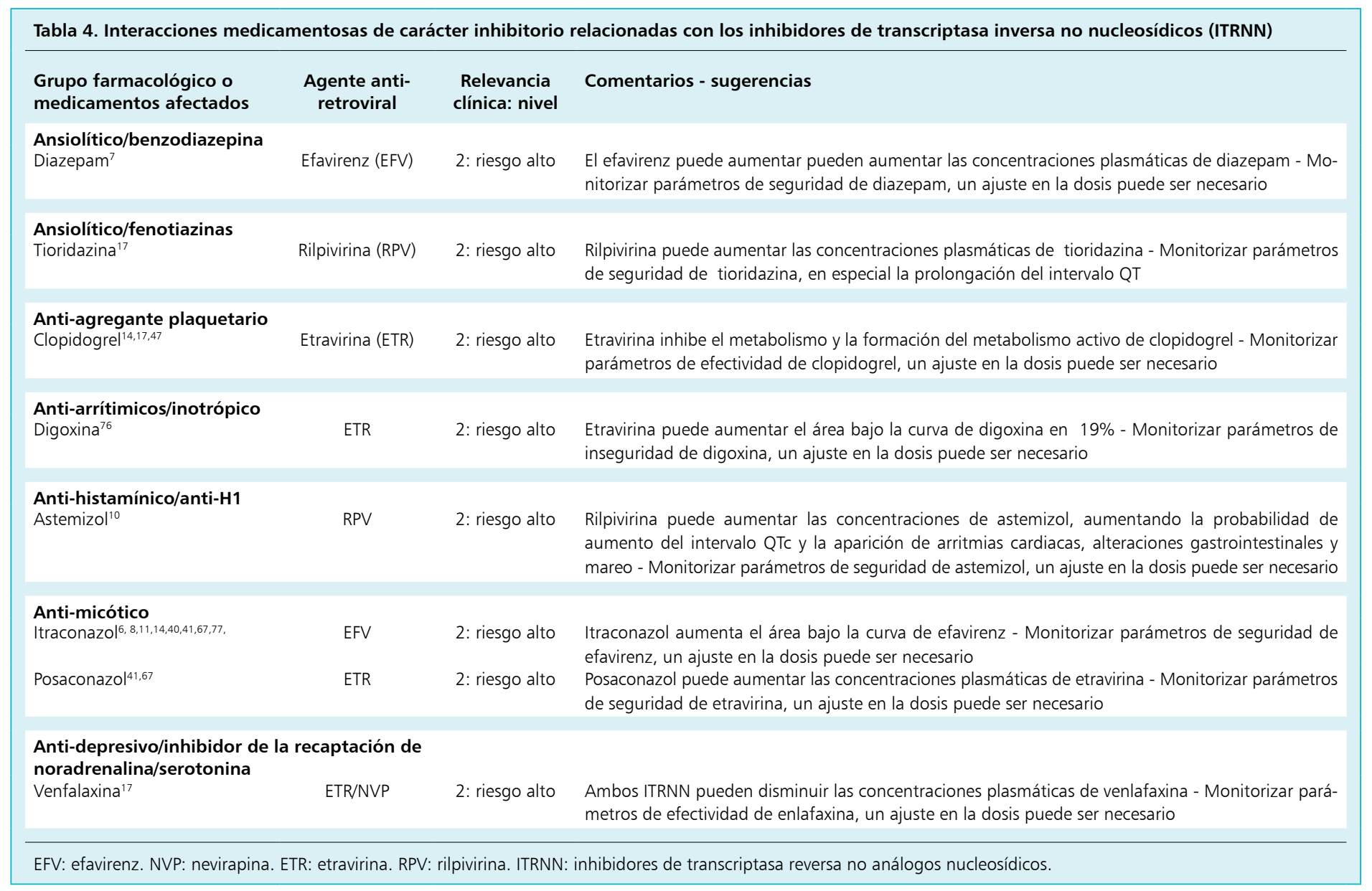

las concentraciones plasmáticas de los IP; mientras que los macrólidos (claritromicina y eritromicina) presentan interacciones bidireccionales de inhibición con los IP (aumento en las concentraciones plasmáticas de los macrólidos y de los inhibidores de proteasa). En la Tabla 3 se presentan las interacciones más relevantes (niveles 1 y 2) relacionadas con IP por inducción enzimática. En algunos casos, los IP pueden disminuir las concentraciones plasmáticas de algunos medicamentos; sin embargo, existen medicamentos y productos naturales, entre ellos rifamicinas y la hierba de San Juan, que son inductores enzimáticos por excelencia, afectando las concentraciones plasmáticas de los IP y pudiendo comprometer la efectividad de los ARV.

En la Tabla 4 se presentan las interacciones más relevantes (niveles 1 y 2) relacionados con los inhibidores de transcriptasa reversa no nucleosídicos (ITRNN) por inhibición enzimática. Por su parte, en la Tabla 5 se presentan las interacciones más relevantes (niveles 1 y 2) relacionadas con los ITRNN por inducción enzimática. En este sentido, en ciertos casos, los ITRNN pueden disminuir las concentraciones plasmáticas de algunos medicamentos. De forma similar, a lo que se identificó con los IP, los antituberculosos, los anticonvulsivantes de primera generación (excepto ácido valproico) y la hierba de San Juan son inductores enzimáticos por excelencia y afectan las concentraciones plasmáticas de los ITRNN y pueden generar problemas de efectividad de estos ARV.

Finalmente, en la Tabla 6 se presentan las interacciones más relevantes (niveles 1 y 2) relacionadas con los inhibidores de transcriptasa reversa análogos nucleosídico (ITRAN), Inhibidores de fusión (IF) e inhibidores de la integrasa (II) por inducción e inhibición enzimática. Al igual que las interacciones más relevantes de carácter farmacodinámico.

Relacionado con el grupo farmacológico ARV relacionado con los 935 pares de IM farmacocinéticas, 511 $(54,7 \%)$ estuvieron asociadas a IP, $202(21,6 \%)$ a ITRNN, $75(8,0 \%)$ con inhibidores de la integrasa, $52(5,6 \%)$ con ITRAN, $58(6,2 \%)$ con el cobicistat (que aunque no es un ARV, es un potenciador farmacocinético) y 37 (4,0\%) con inhibidores de la entrada. 
Tabla 5. Interacciones medicamentosas de carácter inhibitorio relacionadas con los inhibidores de transcriptasa inversa no nucleosídicos (ITINN)

\begin{tabular}{|c|c|c|c|}
\hline $\begin{array}{l}\text { Grupo farmacológico o } \\
\text { medicamentos afectados }\end{array}$ & $\begin{array}{l}\text { Agente anti- } \\
\text { retroviral }\end{array}$ & $\begin{array}{l}\text { Relevancia } \\
\text { clínica: nivel }\end{array}$ & Comentarios - sugerencias \\
\hline \multicolumn{4}{|l|}{ Analgésico opioide } \\
\hline Metadona $51,79-82$ & RPV & 2: riesgo alto & $\begin{array}{l}\text { Rilpivirina puede disminuir el área bajo la curva de la R y S metadona en } 16 \% \text { - Monitorizar paráme- } \\
\text { tros de efectividad de metadona, un ajuste en la dosis puede ser necesario }\end{array}$ \\
\hline
\end{tabular}

\section{Ansiolítico/fenotiazinas}

Tioridazina ${ }^{17}$

EFV/ETR/Nevirapi- $\quad$ 2: riesgo alto

Estos ITRNN pueden disminuir las concentraciones plasmáticas de tioridazina - Monitorizar parámena (NVP)

tros de efectividad de tioridazina, un ajuste en la dosis puede ser necesario

\section{Antimicrobiano/macrólido}

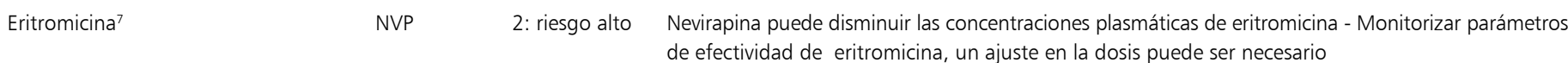

\section{Anti-conceptivo}

Etonogestrel $84-88$

EFV 2: riesgo alto

Efavirenz puede disminuir las concentraciones plasmáticas de etonogestrel y el efecto anticonceptivo se puede ver disminuido - Monitorizar parámetros de efectividad de etonogestrel. Se debe recomendar a la paciente emplear un método barrera como método complementario de planificación

\section{Anti-convulsivantes}

Carbamazepina puede inducir la CYP3A4, aumentar el metabolismo de etravirina y disminuir así sus concentraciones plasmáticas - Monitorizar parámetros de efectividad de etravirina, un ajuste en la dosis puede ser necesario

Fenitoína $6,8,10,11,15,76,89$

ETR 2: riesgo alto

Fenitoína y fenobarbital pueden disminuir las concentraciones plasmáticas de etravirina - Monitorizar

Fenobarbital $6,8,11,15,76,89$ parámetros de efectividad de etravirina, se recomienda utilizar anticonvulsivantes de segunda generación (gabapentina, lamotrigina, vigabatrina y topiramato). Gabapentina al no ser metabolizada por la CYP es considerada la más adecuada

\section{Anti-depresivos atípicos}

Bupropión ${ }^{17}$

ETR 2: riesgo alto

Etravirina puede disminuir las concentraciones plasmáticas de bupropión - Monitorizar parámetros de efectividad de bupropión, un ajuste en la dosis puede ser necesario

Mirtazapina ${ }^{13}$

EFV/ETR/NVP 2: riesgo alto

Estos ITRNN pueden disminuir las concentraciones plasmáticas de mirtazapina - Monitorizar parámetros de efectividad de mirtazapina, un ajuste en la dosis puede ser necesario

Trazodona $^{13} \quad$ EFV/ETR/NVP 2: riesgo al to

Estos ITRNN pueden disminuir las concentraciones plasmáticas de trazodona - Monitorizar parámetros de efectividad de trazodona, un ajuste en la dosis puede ser necesario

Anti-depresivo/inhibidor de la recaptación de noradrenalina/serotonina

2: riesgo alto

Ambos ITRNN pueden disminuir las concentraciones plasmáticas de venlafaxina - Monitorizar parámetros de efectividad de enlafaxina, un ajuste en la dosis puede ser necesario

\section{Anti-depresivo/inhibidor selectivo} de la recaptación de serotonina

NVP $\quad$ 2: riesgo alto

Nevirapina puede disminuir las concentraciones plasmáticas de fluoxetina - Monitorizar parámetros de efectividad de fluoxetina, un ajuste en la dosis puede ser necesario

\section{Anti-diabético/gliptinas}

Linagliptina ${ }^{17}$
EFV/ETR/NVP 2: riesgo alto

Estos ITRNN pueden disminuir las concentraciones plasmáticas de linagliptina - Monitorizar parámetros de efectividad de linagliptina, un ajuste de dosis puede ser necesario

\section{Anti-diabéticos/meglitinidas}

Nateglinida ${ }^{17}$

Repaglinida ${ }^{17}$
EFV/ETR/NVP 2: riesgo alto Estos ITRNN pueden disminuir las concentraciones plasmáticas de nateglinida y repaglinida - Monitorizar parámetros de efectividad de nateglinida y repaglinida, un ajuste en la dosis puede ser necesario 


\begin{tabular}{|c|c|c|c|}
\hline \multicolumn{4}{|l|}{ Continuación Tabla 5} \\
\hline $\begin{array}{l}\text { Grupo farmacológico o } \\
\text { medicamentos afectados }\end{array}$ & $\begin{array}{l}\text { Agente anti- } \\
\text { retroviral }\end{array}$ & $\begin{array}{l}\text { Relevancia } \\
\text { clínica: nivel }\end{array}$ & Comentarios - sugerencias \\
\hline $\begin{array}{l}\text { Anti-hipertensivos/bloqueado } \\
\text { de canales de calcio } \\
\text { Amlodipino }^{14,17,91} \\
\text { Diltiazem }^{14,17,91} \\
\text { Felodipino } \\
\text { Nicardipino } 14,91 \\
\text { Nifedipino }^{14,17,91} \\
\text { Verapamilo }^{14,17,91}\end{array}$ & es & 2: riesgo alto & $\begin{array}{l}\text { Estos ITRNN pueden disminuir las concentraciones plasmáticas de estos antihipertensivos bloquea- } \\
\text { dores de los canales de calcio. Monitorizar parámetros de efectividad de estos medicamentos, un } \\
\text { ajuste en la dosis puede ser necesario }\end{array}$ \\
\hline $\begin{array}{l}\text { Anti-inflamatorio esteroideo } \\
\text { Dexametasona }{ }^{15}\end{array}$ & EFV & 2: riesgo alto & $\begin{array}{l}\text { Dexametasona puede disminuir las concentraciones plasmáticas de efavirenz - Monitorizar paráme- } \\
\text { tros de efectividad de efavirenz, un ajuste en la dosis puede ser necesario }\end{array}$ \\
\hline $\begin{array}{l}\text { Anti-maláricos } \\
\text { Artemeter }{ }^{18,38,65,92-95}\end{array}$ & EFV/NVP & 2: riesgo alto & Ambos ITRNN pueden disminuir las concentraciones plasmáticas de artemeter - Monitorizar pará- \\
\hline Lumefantrina ${ }^{18,38,92-96}$ & EFV/NVP & 2: riesgo alto & $\begin{array}{l}\text { Ambos ITRNN pueden disminuir las concentraciones plasmáticas de lumefantrina - Monitorizar } \\
\text { parámetros de efectividad de lumefantrina, un ajuste en la dosis puede ser necesario }\end{array}$ \\
\hline Quinina $^{97}$ & NVP & 2: riesgo alto & $\begin{array}{l}\text { Nevirapina puede disminuir las concentraciones plasmáticas de quinina - Monitorizar parámetros de } \\
\text { efectividad de quinina, un ajuste en la dosis puede ser necesario }\end{array}$ \\
\hline Amodiaquina $^{39}$ & EFV & 2: riesgo alto & $\begin{array}{l}\text { Efavirenz puede disminuir las concentraciones plasmáticas de amodiaquina - Monitorizar parámetros } \\
\text { de efectividad de amodiaquina, un ajuste en la dosis puede ser necesario }\end{array}$ \\
\hline $\begin{array}{l}\text { Antipsicóticos/atípicos } \\
\text { Quetiapina }^{17} \\
\text { Risperidona }^{17} \\
\text { Ziprasidona }^{17}\end{array}$ & EFV/ETR/NVP & 2: riesgo alto & $\begin{array}{l}\text { Estos ITRNN pueden disminuir las concentraciones plasmáticas de estos antipsicóticos. Monitorizar } \\
\text { parámetros de efectividad de estos medicamentos, un ajuste en la dosis puede ser necesario }\end{array}$ \\
\hline $\begin{array}{l}\text { Antipsicóticos/típicos } \\
\text { Clorpromazina }^{17} \\
\text { Flufenazina }^{17} \\
\text { Haloperidol }^{17} \\
\text { Iloperidona }^{17} \\
\text { Perfenazina }{ }^{17}\end{array}$ & EFV/ETR/NVP & 2: riesgo alto & $\begin{array}{l}\text { Estos ITRNN pueden disminuir las concentraciones plasmáticas de estos antipsicóticos. Monitorizar } \\
\text { parámetros de efectividad de estos medicamentos, un ajuste en la dosis puede ser necesario }\end{array}$ \\
\hline $\begin{array}{l}\text { Antipsicótico/típico } \\
\text { Pimozida17 }\end{array}$ & ETR/NVP & 2: riesgo alto & $\begin{array}{l}\text { Ambos ITRNN pueden disminuir las concentraciones plasmáticas de pimozida - Monitorizar paráme- } \\
\text { tros de efectividad de pimozida, un ajuste en la dosis puede ser necesario }\end{array}$ \\
\hline $\begin{array}{l}\text { Antituberculosos } \\
\text { Bedaquilina }{ }^{98,99,101}\end{array}$ & EFV & 2: riesgo alto & $\begin{array}{l}\text { Efavirenz puede disminuir las concentraciones plasmáticas de bedaquilina - Monitorizar parámetros } \\
\text { de efectividad de bedaquilina, un ajuste en la dosis puede ser necesario. }\end{array}$ \\
\hline Rifampicina $a^{6,8,11,14,63,68-72,74,76,89,101}$ & ETR & 2: riesgo alto & $\begin{array}{l}\text { Rifampicina puede inducir la CYP3A4 y aumentar el metabolismo de etravirina, lo que puede causar } \\
\text { una disminución en sus concentraciones plasmáticas y en la respuesta virológica - Monitorizar pará- } \\
\text { metros de efectividad de etravirina, un ajuste en la dosis puede ser necesario }\end{array}$ \\
\hline \multicolumn{4}{|l|}{$\begin{array}{l}\text { Anti-ulceroso/inhibidor de } \\
\text { bomba de protones }\end{array}$} \\
\hline Omeprazol $^{102}$ & EFV & 2: riesgo alto & $\begin{array}{l}\text { Efavirenz puede disminuir las concentraciones plasmáticas de omeprazol - Monitorizar parámetros } \\
\text { de efectividad de omeprazol, un ajuste en la dosis puede ser necesario }\end{array}$ \\
\hline \multicolumn{4}{|l|}{ Hipolipemiantes/estatinas } \\
\hline Rosuvastatina ${ }^{8}$ & EFV & 2: riesgo alto & $\begin{array}{l}\text { Efavirenz puede disminuir las concentraciones plasmáticas de rosuvastatina - Monitorizar paráme- } \\
\text { tros de efectividad de rosuvastatina, un ajuste en la dosis puede ser necesario según perfil lipídico. }\end{array}$ \\
\hline Simvastatina ${ }^{17,47,76,103}$ & ETR & 2: riesgo alto & $\begin{array}{l}\text { Etravirina puede disminuir las concentraciones plasmáticas de simvastatina - Monitorizar parámetros } \\
\text { de efectividad de simvastatina, un ajuste en la dosis puede ser necesario según sea el perfil lipídico }\end{array}$ \\
\hline \multicolumn{4}{|l|}{ Inmunosupresor } \\
\hline Sirolimus $4^{48}$ & EFV & 2: riesgo alto & $\begin{array}{l}\text { Efavirenz puede disminuir las concentraciones plasmáticas de sirolimus - Monitorizar los parámetros } \\
\text { de efectividad de sirolimus, un ajuste en la dosis puede ser necesario }\end{array}$ \\
\hline \multicolumn{4}{|l|}{ Fitoterapéutico } \\
\hline Hierba de San Juan ${ }^{6,8,11,76}$ & ETRV & 2: riesgo alto & $\begin{array}{l}\text { La hierba de San Juan puede inducir la CYP3A4 y aumentar el metabolismo de etravirina, lo que } \\
\text { puede causar una disminución en sus concentraciones plasmáticas y en la respuesta virológica - Mo- } \\
\text { nitorizar parámetros de efectividad de etravirina, un ajuste en la dosis puede ser necesario }\end{array}$ \\
\hline
\end{tabular}




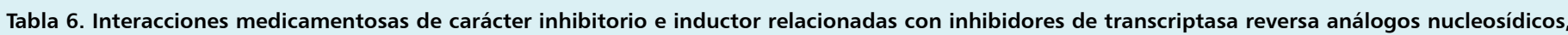
inhibidores de fusión e inhibidores de integrasa; además interacciones más relevantes de mecanismo farmacodinámico

\begin{tabular}{|c|c|c|c|}
\hline $\begin{array}{l}\text { Grupo farmacológico o } \\
\text { medicamentos afectados }\end{array}$ & $\begin{array}{l}\text { Agente anti- } \\
\text { retroviral }\end{array}$ & $\begin{array}{l}\text { Relevancia } \\
\text { clínica: nivel }\end{array}$ & Comentarios - sugerencias \\
\hline $\begin{array}{l}\text { Analgésico opioide } \\
\text { Metadona } 11,13,51,53,54\end{array}$ & Zidovudina (AZT) & 2: riesgo alto & $\begin{array}{l}\text { Metadona puede aumentar el área bajo la curva de zidovudina en } 40 \% \text { - Monitorizar parámetros } \\
\text { de seguridad de zidovudina, un ajuste en la dosis puede ser necesario }\end{array}$ \\
\hline $\begin{array}{l}\text { Antiagregante plaquetario } \\
\text { Ácido acetil salicílico (AAS) }{ }^{103}\end{array}$ & Tenofovir (TDF) & 2: riesgo alto & $\begin{array}{l}\text { Tenofovir y AAS compiten por eliminación renal y pueden aumentar sus concentraciones plasmá- } \\
\text { ticas - Monitorizar parámetros de seguridad de tenofovir y AAS, un ajuste en la dosis puede ser } \\
\text { necesario }\end{array}$ \\
\hline
\end{tabular}

\section{Antidepresivo atípico}

Nefazodona ${ }^{17} \quad$ Maraviroc 2: riesgo alto

Maraviroc puede aumentar las concentraciones plasmáticas de nefazodona - Monitorizar parámetros de seguridad de nefazodona, una disminución de la dosis de nefazodona a 150 mg puede ser necesario

\section{Antimicótico}

Anfotericina $B^{7,41}$

Fluconazol $7,14,41$

Ketoconazol ${ }^{7}$

Fluconazol7,14,41

Voriconazol ${ }^{41,67}$

2: riesgo alto

Flucitosina ${ }^{4}$

AZT

2: riesgo alto

de seguridad de maraviroc, un ajuste en la dosis puede ser necesario

\section{Antituberculoso}

Rifampicina $8,11,60,63,68-72,74,101,104-113 \quad$ Abacavir $(A B C) /$

Maraviroc/Ralte-

gravir (RAL)

\section{Antiviral}

Ribavirina ${ }^{114}$

TDF

2: riesgo alto

El uso concomitante de zidovudina y flucitosina puede generar depresión de medula ósea - Monitorizar parámetros o signos de toxicidad medular

Rifampicina puede disminuir las concentraciones plasmáticas de abacavir entre 40 y $60 \%$, al igua que las concentraciones de maraviroc y raltegravir - Monitorizar parámetros de efectividad de abacavir, maraviroc y raltegravir, un ajuste en la dosis puede ser necesario; para raltegravir se recomienda ajustar a $800 \mathrm{mg}$ cada $12 \mathrm{~h}$

\section{ARV/inhibidor de fusión}

Maraviroc ${ }^{118}$

Tenofovir y ribavirina compiten por eliminación renal y pueden aumentar sus concentraciones plasmáticas - Monitorizar parámetros de seguridad de tenofovir y ribavirina, un ajuste en la dosis puede ser necesario

\section{ARV/inhibidor de proteasa}

Darunavir88 805,106

RAL 2: riesgo alto

Elvitegravir puede aumentar las concentraciones plasmáticas de maraviroc - Monitorizar parámetros de seguridad de maraviroc, un ajuste en la dosis puede ser necesario

ARV/inhibidor de transcriptasa

inversa no nucleosídico

Etravirina ${ }^{116,115,117}$

Dolutegravir 2: riesgo alto

(DTG) /Maraviroc

Etravirina puede disminuir las concentraciones plasmáticas de dolutegravir, mientras que maraviroc puede disminuir las concentraciones plasmáticas de etravirina - Monitorizar parámetros de efectividad de dolutegravir o etravirina según el caso, un ajuste en la dosis puede ser necesario

\section{Fitoterapéutico}

Panax ginseng 121

RAL

2: riesgo alto

Raltegravir puede disminuir las concentraciones plasmáticas de darunavir - Monitorizar parámetros de efectividad de darunavir, se recomienda el uso de darunavir con ritonavir

\section{Vitaminas}

Vitamina D3 ${ }^{122}$

TDF

2: riesgo alto

\section{Antiasmático}

Montelukast ${ }^{123}$

2: riesgo alto

Panax ginseng puede aumentar las concentraciones plasmáticas de raltegravir - Monitorizar parámetros de seguridad de raltegravir, un ajuste de dosis puede ser necesario

Tenofovir y la vitamina D3 compiten por eliminación renal y pueden aumentar sus concentraciones plasmáticas - Monitorizar parámetros de seguridad de tenofovir y vitamina D3, un ajuste en la dosis puede ser necesario 


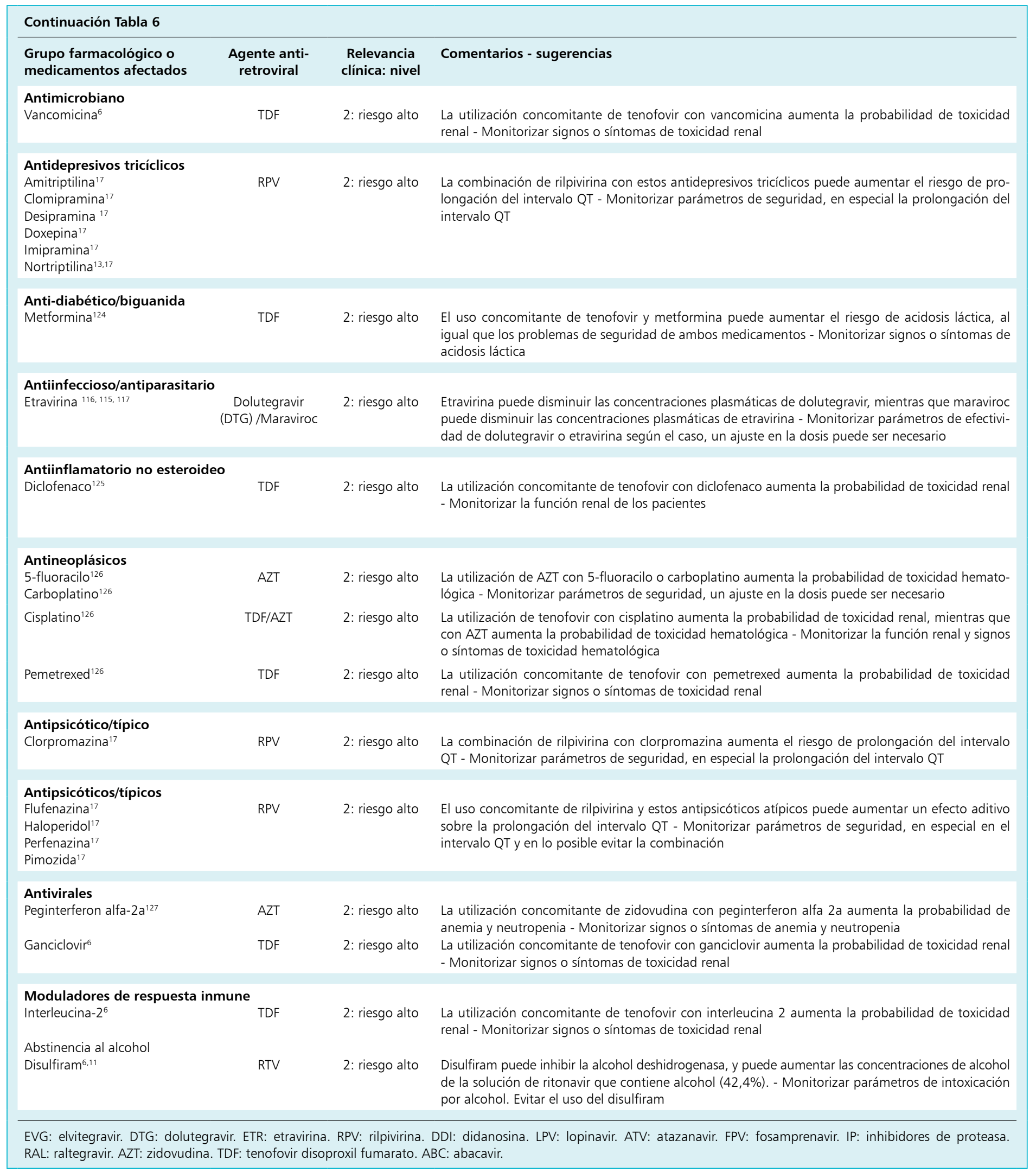


En la búsqueda también se identificaron 434 parejas de interacciones de medicamentos con nelfinavir (n: 81), saquinavir (n: 84), amprenavir (n: 72), tipranavir (n: 85), indinavir (n: 86), delavirdina (n: 8), estavudina (n: 13), y lersivirina (n: 5). Los cuales no se incluyeron, debido a que estos medicamentos ya no se emplean o están en desuso en la práctica clínica. De estas 434 interacciones no incluidas en el presente trabajo, 271 son de nivel 1 y 2 niveles de mayor relevancia clínica en caso de emplearse estos medicamentos.

\section{Discusión}

Este trabajo presenta los resultados de la tercera revisión estructurada de IM de ARV, en el cual se identificaron 935 parejas nuevas de IM, incluyendo las que pueden presentar algunos ARV de más reciente comercialización, como son dolutegravir y elvitegravir, y algunos potenciadores farmacocinéticos como cobicistat. Por ello, estos resultados pueden facilitar la tarea clínica de evaluar y establecer el efecto de las IM en la farmacoterapia y resultados en salud de pacientes con VIH/SIDA.

De las 935 parejas de IM, 895 (95,7\%) fueron explicadas por mecanismos farmacocinéticos, de las cuales 561 se atribuyen a inhibición enzimática. Este resultado, es similar al 95\% establecido en las revisiones previas ${ }^{1,2}$ y soporta la capacidad que tienen los ARV de modificar o verse afectados no sólo por la actividad de la CYP450, sino también, por otras estructuras que tienen la capacidad de alterar la biodisponibilidad de los fármacos, como lo es la glicoproteína-P (Gp-P). Por ello, se podría establecer que, en pacientes con TARV, la determinación y evaluación de la relevancia clínica de una interacción farmacocinética se fundamenta en el entendimiento y en la utilización de los conceptos relacionados con la estimulación y, especialmente, con la inhibición enzimática (fundamentalmente de las subfamilias CYP3 y CYP2) y, en menor medida, con la absorción (especialmente con cambios en el metabolismo pre-sistémico del fármaco y/o en la actividad transportadora de la Gp-P).

De las 935 parejas de IM, 402 (43\%) fueron de relevancia clínica (niveles 1 y 2) y, por tanto, con estas combinaciones existe la probabilidad de presentar un mayor riesgo de interacción y que éste afecte la efectividad o la seguridad de la farmacoterapia y, con ello, los resultados en salud en pacientes con VIH/SIDA. Sin embargo, la información de la relevancia clínica de la IM debe ser valorada en el contexto de la experiencia clínica del prescriptor, quien por las características clínicas del paciente podría tomar el juicio clínico de utilizar medicamentos con una probabilidad de ocurrencia y gravedad nivel 1 (contraindicación de la combinación). Lo importante es que en estos casos, al igual que en las de nivel 2, será necesario un seguimiento más cercano de los resultados en salud, tanto en términos de efectividad (respuesta inmunológica y virológica) como de seguridad (parámetros clínicos asociados a la presentación de los eventos adversos) con la TARV.

En la presente revisión se evidenció una disminución significativa en el porcentaje de IM de nivel 1, el que disminuyó de $6 \%$ en las revisiones previas ${ }^{1,2}$ a $0,43 \%$. Este resultado podría, entre otras, ser explicado por algunas hipótesis: 1) En el diseño y desarrollo de los nuevos ARV, se están teniendo en cuenta este factor, generando fármacos con menos probabilidad de interacciones $^{131,132}$; 2) El retiro del mercado de medicamentos relacionados con efectos adversos e interacciones de riesgo muy alto, como terfenadina y cerivastatina ${ }^{132}$; y 3 ) Algunos de los ARV responsables de interacciones han empezado a ser utilizados recientemente, dolutegravir (año 2013) y elvitegravir (año 2013) y, por tanto, aún no se han documentado las experiencias clínicas referentes a información sobre sus interacciones medicamentosas y el efecto en el paciente.

La propuesta de clasificación de la relevancia clínica de las $\mathrm{IM}^{4}$, utilizada en la presente revisión, es la misma empleada en trabajos previos ${ }^{1-3} \mathrm{y}$, aunque ha mostrado ser funcional para establecer y utilizar la relevancia clínica de las interacciones encontradas, se requiere de trabajos que evidencien su utilidad en la práctica clínica. En este contexto, las 935 parejas de IM identificadas serán el soporte para actualizar el Software para el análisis de la relevancia clínica de las IM con ARV (SIMARV®), disponible en la página del grupo de promoción y prevención farmacéutica de la Universidad de Antioquia, Medellín-Colombia, lo que facilita que se valore la funcionalidad de la propuesta e interacciones identificadas. Además su acceso es libre y se puede consultar en la página Web http://us134078.bizhostnet.com/softwareim/ Inicio/Default.aspx

Debido a la importancia que este tipo de programas, en el logro de la finalidad de facilitar y optimizar la detección de interacciones medicamentosas de relevancia clínica, en el momento de la prescripción médica, se recomiendan, además del link de SIMARV®, la consulta de páginas web como: http://www.hiv-druginteractions. org/; http://www.medinteract.net/ y https://www.drugs. com/drug_interactions.html.

\section{Limitaciones}

Las limitación principal de este estudio es que la búsqueda se restringió a la base de datos MEDLINE/ PubMed y de la estrategia de búsqueda aunque se tenía como filtro textos completos, no todos cumplieron con este requisito. Sin embargo, este efecto podría haber sido minimizado, debido a que la revisión se complementó 
con la revisión e inclusión de referencias bibliográficas consideradas como relevantes de los artículos identificados en la búsqueda.

\section{Conclusiones - comentarios finales}

En esta revisión estructurada de IM de ARV, se identificaron 935 nuevas parejas de IM, principalmente interacciones medicamento/medicamento: 861 (92\%) y, en menor medida, a interacciones de medicamentos con fitoterapéuticos, enfermedades, condiciones especiales, alimentos o drogas de abuso: 74 (8\%).

El mecanismo que explica la aparición en la de las 935 parejas de IM es farmacocinético: 895 (95,7\%), especialmente inhibición enzimática: 561 (60\%). En el caso de las 40 (4,3\%) IM atribuidas a mecanismo farmacodinámico, la mayoría se debe por sinergismo. Relacionado con el nivel de relevancia clínica, 402 (43\%) parejas de IM presentan un mayor riesgo de generar problemas de efectividad y seguridad de los medicamentos ARV: 4 $(0,43 \%)$ de nivel 1 y $398(42,6 \%)$ de nivel 2 .

Debido a que la terapia ARV genera un número importante de interacciones de relevancia clínica, es necesario continuar revisando y sistematizando la información que se publique sobre este tema, evaluando su evidencia y estableciendo su relevancia clínica. Además, se requiere del diseño de estudios para valorar la utilidad y funcionalidad de la propuesta para identificar y establecer la relevancia clínica de las IM en pacientes con VIH/SIDA.

\section{Resumen}

Objetivo: Actualizar información sobre interacciones medicamentosas en pacientes con VIH/SIDA. Métodos: Revisión estructurada en MEDLINE/PubMed utilizando los términos Mesh: Anti-retroviral agents and drug interactions or herb-drug interactions or food-drug interactions, entre mayo de 2009 y diciembre de 2014. Publicaciones sobre interacciones medicamentosas, en humanos, en inglés o español y con acceso a texto completo fueron seleccionadas. Además, se incluyeron referencias de artículos considerados relevantes. La inclusión de los artículos fue evaluada por tres investigadores independientes y, en caso de requerirlo, por consenso entre ellos. La relevancia clínica se estableció en cuatro niveles, acorde con la gravedad y probabilidad de ocurrencia de la interacción. Resultados: Se identificaron 546 artículos, de los cuales se seleccionaron 273; además, se incluyeron 11 referencias relevantes. Se identificaron 935 parejas de interacciones medicamentosas, 95,7\% farmacocinéticas. De este grupo, 823 mediadas por inducción o inhibición enzimática y 67 por cambios en la biodisponibilidad. De las 935 parejas de interacciones, 402 (43\%) fueron relevantes clínicamente (niveles 1 o 2). Conclusiones: Las interacciones medicamentosas con anti-retrovirales de mayor relevancia clínica se deben a mecanismos farmacocinéticos, principalmente inducción o inhibición enzimática. Acorde con revisiones previas, los inhibidores de proteasa continúan siendo los anti-retrovirales con mayor número de interacciones relevantes.

\section{Referencias bibliográficas}

1.- Amariles P, Giraldo N A, Faus M J. Interacciones medicamentosas en pacientes infectados con el VIH: aproximación para establecer y evaluar su relevancia clínica. Farm Hosp 2007; 31 (5): 283-302.

2.- Giraldo N A, Amariles P, Gutiérrez FJ, Monsalve M, Faus M J. Aproximación para establecer y evaluar la relevancia clínica de las interacciones medicamentosas en pacientes infectados con virus de la inmunodeficiencia humana: actualización 2009. Farm Hosp 2010; 34 (2): 90-3.

3.- Amariles P, Giraldo N A, Faus M J. Clinical relevance of drug interactions in HIV-infected patients receiving antiretroviral therapy. In: Kasenga F (Ed). Understanding HIV/AIDS Management and Care - Pandemic Approaches in the 21 st Century. InTech, Croatia. 2011. p 301-58.

4.- Amariles P, Giraldo N A, Faus M J. Interacciones medicamentosas: aproximación para establecer y evaluar su relevancia clínica.
Med Clin (Barc) 2007; 129 (1): 27-35.

5.- van Waterschoot R A, ter Heine R, Wagenaar E, van der Kruijssen C M, Rooswinkel R W, Huitema A D et al. Effects of cytochrome P450 3A (CYP3A) and the drug transporters P-glycoprotein (MDR1/ABCB1) and MRP2 (ABCC2) on the pharmacokinetics of lopinavir. Br J Pharmacol 2010; 160 (5): 1224-33.

6.- Panel de expertos de Gesida y Plan Nacional sobre el SIDA. [Consensus document of Gesida and Spanish Secretariat for the National Plan on AIDS (SPNS) regarding combined antiretroviral treatment in adults infected by the human immunodeficiency virus (January 2012)]. Enferm Infecc Microbiol Clin 2012; 30 (6): e1-89.

7.- Gonçalves L S, Gonçalves B M, de Andrade M A, Alves F R, Junior A S. Drug interactions during periodontal therapy in $\mathrm{HIV}$-infected subjects. Mini Rev Med Chem 2010; 10 (8): 766-72.

8.- Ceballos M E. Considerations in first line antiretroviral selection for adults. Rev Chilena Infectol 2013; 30 (5): 522-37.
9.- Deems ED. Darunavir: a review of its use in the management of HIV-1 infection. Drugs 2014; 74 (1): 99-125.

10.- Tan D H, Walmsley S L. Management of persons infected with human immunodeficiency virus requiring admission to the intensive care unit. Crit Care Clin 2013; 29 (3): 603-20.

11.- Expert Panel of GESIDA and the National AIDS Plan. Executive summary of the GESIDA/ National AIDS Plan Consensus Document on antiretroviral therapy in adults infected by the human immunodeficiency virus (updated January 2015). Enferm Infecc Microbiol Clin 2015; 33 (8): 544-56.

12.- Hsu A J, Carson K A, Yung R, Pham P A. Severe prolonged sedation associated with co administration of protease inhibitors and intravenous midazolam during bronchoscopy. Pharmacotherapy 2012; 32 (6): 538-45.

13.- Gallego L, Barreiro P, López-Ibor J J. Psychopharmacological treatments in HIV patients under antiretroviral therapy. AIDS Rev 2012; 14 (2): 101-11.

14.- Lauren J Gleason, Amneris E Luque, Krupa 
Shah. Polypharmacy in the HIV-infected older adult population. Clin Interv Aging 2013; 8: 749-63.

15.- Ganesan A, Masur H. Critical care of persons infected with the human immunodeficiency virus. Clin Chest Med 2013; 34 (2): 307-23.

16.- Tseng A, Szadkowski L, Walmsley S, Salit I, Raboud J. Association of age with polypharmacy and risk of drug interactions with antiretroviral medications in HIV-positive patients. Ann Pharmacother 2013; 47 (11): 1429-39.

17.- Nachega J B, Hsu A J, Uthman O A, Spinewine A, Pham P A. Antiretroviral therapy adherence and drug-drug interactions in the aging HIV population. AIDS 2012; 26 (Suppl 1): S39-53.

18.- Panel de expertos de Gesida, Secretaría del Plan Nacional sobre el SIDA (SPNS) y Asociación Española para el Estudio del Hígado (AEEH). Recommendations of Gesida/PNS/AEEH for the management and treatment of the adult patient co-infected with HIV and hepatitis A, B and C virus. Enferm Infecc Microbiol Clin 2010; 28 (1): 31e1-31e31

19.- Croom K F, Dhillon S, Keam S J. Atazanavir: a review of its use in the management of HIV-1 infection. Drugs 2009; 69 (8): 1107-40.

20.- Hall J J, Hughes C A, Foisy M M, Houston S, Shafran S. Iatrogenic Cushing syndrome after intra-articular triamcinolone in a patient receiving ritonavir-boosted darunavir. Int J STD AIDS 2013; 24 (9): 748-52.

21.- Gray D, Roux P, Carrihill M, Klein M. Adrenal suppression and Cushing's syndrome secondary to ritonavir and budesonide. S Afr Med J 2010; 100 (5): 296-7.

22.- Blondin M C, Beauregard H, Serri O. Iatrogenic Cushing syndrome in patients receiving inhaled budesonide and itraconazole or ritonavir: two cases and literature review. Endocr Pract 2013; 19 (6): e138-41.

23.- Saberi P, Phengrasamy T, Nguyen D P. Inhaled corticosteroid use in HIV-positive individuals taking protease inhibitors: a review of pharmacokinetics, case reports and clinical management. HIV Med 2013; 14 (9): 519-29.

24.- Canalejo E, Pacheco MS. Cushing syndrome due to ritonavir-fluticasone interaction. Can Med Assoc J 2012; 184 (15): 1714.

25.- Daveluy A, Raignoux C, Miremont-Salamé G, Girodet P O, Moore N, Haramburu F, et al. Drug interactions between inhaled corticosteroids and enzymatic inhibitors. Eur J Clin Pharmacol 2009; 65 (7): 743-5.

26.- St Clair K, Maguire J D. Role of fluconazole in a case of rapid onset ritonavir and inhaled fluticasone-associated secondary adrenal insufficiency. Int J STD AIDS 2012; 23 (5): 371-2.

27.- Perry M E, Almaani N, Desai N, Larbalestier N, Fox J, Chilton D. Raltegravir-induced Drug Reaction with Eosinophilia and Systemic Symptoms (DRESS) syndrome - implications for clinical practice and patient safety. Int J STD AIDS 2013; 24 (8): 639-42.

28.- Albert N E, Kazi S, Santoro J, Dougherty R.
Ritonavir and epidural triamcinolone as a cause of iatrogenic Cushing's syndrome. Am J Med Sci 2012; 344 (1): 72-4

29.- Hall J J, Hughes C A, Foisy M M, Houston S, Shafran S. Iatrogenic Cushing syndrome after intra-articular triamcinolone in a patient receiving ritonavir-boosted darunavir. Int J STD AIDS 2013; 24 (9): 748-52.

30.- Abgrall S, Le Bel J, Lele N, Laouénan C, Eychenne N, Mentré F, et al. Lack of effect of doxycycline on trough concentrations of protease inhibitors or non-nucleoside reverse transcriptase inhibitors in $\mathrm{HIV}$-infected patients. HIV Clin Trials 2013; 14 (6): 313-8.

31.- Lakatos B, Stoeckle M, Elzi L, Battegay M, Marzolini C. Gastrointestinal bleeding associated with rivaroxaban administration in a treated patient infected with human immunodeficiency virus. Swiss Med Wkly 2014; 144: w13906.

32.- Vieira C S, Bahamondes M V, de Souza R M, Brito M B, Rocha Prandini T R, Amaral E, et al. Effect of antiretroviral therapy including lopinavir/ritonavir or efavirenz on etonogestrelreleasing implant pharmacokinetics in HIVpositive women. J Acquir Immune Defic Syndr 2014; 66 (4): 378-85.

33.- Hill L, Lee K C. Pharmacotherapy considerations in patients with HIV and psychiatric disorders: focus on antidepressants and antipsychotics. Ann Pharmacother 2013; 47 (1): 75-89.

34.- Jann MW, Spratlin V, Momary K, Zhang H, Turner D, Penzak SR, et al. Lack of a pharmacokinetic drug-drug interaction with venlafaxine extended-release/indinavir and desvenlafaxine extended-release/indinavir. Eur J Clin Pharmacol 2012; 68 (5): 715-21.

35.- Byakika-Kibwika P, Lamorde M, OkabaKayom V, Mayanja-Kizza H, Katabira E, Hanpithakpong W, et al. Lopinavir/ritonavir significantly influences pharmacokinetic exposure of artemether/lumefantrine in HIV-infected Ugandan adults. J Antimicrob Chemother 2012; 67 (5): 1217-23.

36.- Heidari S, Mofenson L M, Hobbs C V, Cotton M F, Marlink R, Katabira E. Unresolved antiretroviral treatment management issues in HIV-infected children. J Acquir Immune Defic Syndr 2012; 59 (2): 161-9.

37.- Van Geertruyden J P, Colebunders R, D'alessandro U. HIV-1-infected individuals traveling to malaria endemic areas. J Acquir Immune Defic Syndr 2009; 51 (2): 34-5.

38.- Kiang T K, Wilby K J, Ensom M H. Clinical pharmacokinetic drug interactions associated with artemisinin derivatives and HIV-antivirals. Clin Pharmacokinet 2014; 53 (2): 141-53.

39.- González R, Ataíde R, Naniche D, Menéndez C, Mayor A. HIV and malaria interactions: where do we stand? Expert Rev Anti Infect Ther 2012; 10 (2): 153-65.

40.- Krishna G, Moton A, Ma L, Martinho M, Seiberling M, McLeod J. Effects of oral posaconazole on the pharmacokinetics of atazanavir alone and with ritonavir or with efavirenz in healthy adult volunteers. J Acquir Immune Defic Syndr 2009; 51 (4): 437-44.

41.- Hughes CA, Foisy M, Tseng A. Interactions between antifungal and antiretroviral agents. Expert Opin Drug Saf 2010; 9 (5): 723-42.

42.- Avihingsanon A, Ramautarsing RA Suwanpimolkul G, Chetchotisakd P, Bowonwatanuwong C, Jirajariyavej S, et al. Ergotism in Thailand caused by increased access to antiretroviral drugs: a global warning. Top Antivir Med 2014; 21 (5): 165-8.

43.- Levêque D, Santucci R, Pavillet J, Herbrecht R, Bergerat J P. Paralytic ileus possibly associated with interaction between ritonavir/lopinavir and vincristine. Pharm World Sci 2009; 31 (6): 619-21.

44.- Vaccher E, Spina M, di Gennaro G, Talamini R, Nasti G, Schioppa O, et al. Concomitant cyclophosphamide, doxorubicin, vincristine, and prednisone chemotherapy plus highly active antiretroviral therapy in patients with human immunodeficiency virus-related, non Hodgkin lymphoma. Cancer 2001; 91 (1): 155-63.

45.- Deeks E D. Atazanavir: in pediatric patients with HIV-1 infection. Paediatr Drugs 2012; 14 (2): 131-41.

46.- Fisher S D, Kanda B S, Miller T L, Lipshultz S E. Cardiovascular disease and therapeutic drug-related cardiovascular consequences in HIV-infected patients. Am J Cardiovasc Drugs 2011; 11 (6): 383-94.

47.- Gedela K, Vibhuti M, Pozniak A, Ward B, Boffito M. Pharmacological management of cardiovascular conditions and diabetes in older adults with HIV infection. HIV Med 2014; 15 (5): 257-68.

48.- Dinavahi R V, Mehrotra A, Murphy B T, Huprikar S. Human immunodeficiency virus and renal transplantation. Kidney Int 2009; 76 (8): 907-10.

49.- Pal D, Kwatra D, Minocha M, Paturi D K, Budda B, Mitra A K. Efflux transporters- and cytochrome $\mathrm{P}-450$-mediated interactions between drugs of abuse and antiretrovirals. Life Sci 2011; 88 (21-22): 959-71.

50.- Papaseit E, Vázquez A, Pérez-Mañá C, Pujadas M, de la Torre R, Farré M, et al. Surviving life-threatening MDMA (3,4-methylenedioxymethamphetamine, ecstasy) toxicity caused by ritonavir (RTV). Intensive Care Med 2012; 38 (7): 1239-40.

51.- Bruce R D, Moody D E, Altice F L, Gourevitch M N, Friedland G H. A review of pharmacological interactions between HIV or hepatitis $\mathrm{C}$ virus medications and opioid agonist therapy: implications and management for clinical practice. Expert Rev Clin Pharmacol 2013; 6 (3): 249-69.

52.- Khalsa J H, Elkashef A. Drug interactions between antiretroviral medications and medications used in the treatment of drug addiction: research needs. Am J Addict 2010; 19 (1): 96-100.

53.- Gruber V A, McCance-Katz E F. Methadone, buprenorphine, and street drug interactions with antiretroviral medications. Curr HIV/AIDS Rep 2010; 7 (3): 152-60. 
54.- Altice F L, Kamarulzaman A, Soriano V V, Schechter M, Friedland G H. Treatment of medical, psychiatric, and substance-use comorbidities in people infected with HIV who use drugs. Lancet 2010; 376 (9738): 367-87.

55.- Gruber V A, Rainey P M, Moody D E, Morse G D, Ma Q, Prathikanti S, et al. Interactions between buprenorphine and the protease inhibitors darunavir-ritonavir and fosamprenavir-ritonavir. Clin Infect Dis 2012; 54 (3): 414-23.

56.- Wire M B, McLean H B, Pendry C, Theodore D, Park J W, Peng B. Assessment of the pharmacokinetic interaction between eltrombopag and lopinavir-ritonavir in healthy adult subjects. Antimicrob Agents Chemother 2012; 56 (6): 2846-51.

57.- Robinson J A, Jamshidi R, Burke A E. Contraception for the HIV-positive woman: a review of interactions between hormonal contraception and antiretroviral therapy. Infect Dis Obstet Gynecol 2012; 2012: 890160.

58.- Ruela Corrêa J C, D'Arcy D M, dos Reis Serra C H, Nunes Salgado H R. Darunavir: a critical review of its properties, use and drug interactions. Pharmacology 2012; 90 (1-2): 102-9.

59.- Tseng A, Hills-Nieminen C. Drug interactions between antiretrovirals and hormonal contraceptives. Expert Opin Drug Metab Toxicol 2013; 9 (5): 559-72.

60.- Dickinson L, Khoo S, Back D. Pharmacokinetics and drug-drug interactions of antiretrovirals: an update. Antiviral Res 2010; 85 (1): 176-89.

61.- Agboghoroma C O. Contraception in the context of HIV/AIDS: a review. Afr J Reprod Health 2011; 15 (3): 15-23.

62.- Schöller-Gyüre M, Kakuda T N, Woodfall B, Aharchi F, Peeters M, Vandermeulen K, et al. Effect of steady-state etravirine on the pharmacokinetics and pharmacodynamics of ethinylestradiol and norethindrone. Contraception 2009; 80 (1): 44-52.

63.- Brown K C, Paul S, Kashuba A D. Drug interactions with new and investigational antiretrovirals. Clin Pharmacokinet 2009; 48(4): 211-41.

64.- Lorenzini K I, Calmy A, Ambrosioni J, Assouline B, Daali Y, Fathi M, et al. Serotonin syndrome following drug-drug interactions and CYP2D6 and CYP2C19 genetic polymorphisms in an HIV-infected patient. AIDS 2012; 26 (18): 2417-8.

65.- Kakuda T N, DeMasi R, van Delft Y, Mohammed P. Pharmacokinetic interaction between etravirine or darunavir/ritonavir and artemether/lumefantrine in healthy volunteers: a two-panel, two-way, two-period, randomized trial. HIV Med 2013; 14 (7): 421-9.

66.- Brüggemann R J, van Luin M, Colbers E P, van den Dungen M W, Pharo C, Schouwenberg $\mathrm{B} \mathrm{J}$, et al. Effect of posaconazole on the pharmacokinetics of fosamprenavir and vice versa in healthy volunteers. J Antimicrob Chemother 2010; 65 (10): 2188-94.

67.- Vadlapatla R K, Patel M, Paturi D K, Pal D,
Mitra A K. Clinically relevant drug-drug interactions between antiretrovirals and antifungals. Expert Opin Drug Metab Toxicol 2014; 10 (4): 561-80.

68.- Gray J M, Cohn D L. Tuberculosis and HIV coinfection. Semin Respir Crit Care Med 2013; 34 (1): 32-43.

69.- Nachega J B, Rosenkranz B, Simon G, Chaisson R E, Diacon A, Taljaard J. Management of adult active tuberculosis disease in era of HIV pandemic, current practices and future perspectives. Infect Disord Drug Targets 2011; 11 (2): 134-43.

70.- Sterling T R, Pham P A, Chaisson R E. HIV infection-related tuberculosis: clinical manifestations and treatment. Clin Infect Dis 2010; 50 (Suppl 3): S223-30.

71.- Anandaiah A, Dheda K, Keane J, Koziel H, Moore D A, Patel N R. Novel developments in the epidemic of human immunodeficiency virus and tuberculosis coinfection. Am J Respir Crit Care Med 2011; 183 (8): 987-97.

72.- Gengiah T N, Gray A L, Naidoo K, Karim Q A. Initiating antiretrovirals during tuberculosis treatment: a drug safety review. Expert Opin Drug Saf 2011; 10 (4): 559-74.

73.- Venkatesh K K, Swaminathan S, Andrews J R, Mayer K H. Tuberculosis and HIV co-infection: screening and treatment strategies. Drugs 2011; 71 (9): 1133-52.

74.- Schutz C, Meintjes G, Almajid F, Wilkinson R J, Pozniak A. Clinical management of tuberculosis and HIV-1 co-infection. Eur Respir J 2010; 36 (6): $1460-81$.

75.- Busse K H, Hadigan C, Chairez C, Alfaro R M, Formentini E, Kovacs J A, et al. Gemfibrozil concentrations are significantly decreased in the presence of lopinavir-ritonavir. J Acquir Immune Defic Syndr 2009; 52(2): 235-9.

76.- Kakuda T N, Schöller-Gyüre M, Hoetelmans R M. Pharmacokinetic interactions between etravirine and non-antiretroviral drugs. Clin Pharmacokinet 2011; 50 (1): 25-39.

77.- Rakhmanina N Y, van den Anker J N. Efavirenz in the therapy of HIV infection. Expert Opin Drug Metab Toxicol 2010; 6 (1): 95-103.

78.- Langdon G, Davis J, Layton G, Chong C L, Weissgerber G, Vourvahis M. Effects of ketoconazole and valproic acid on the pharmacokinetics of the next generation NNRTI, lersivirine (UK-453,061), in healthy adult subjects. Br J Clin Pharmacol 2012; 73(5): 768-75.

79.- Sharma M, Saravolatz L D. Rilpivirine: a new non-nucleoside reverse transcriptase inhibitor J Antimicrob Chemother 2013; 68 (2): 250-6.

80.- Crauwels H, van Heeswijk RP, Stevens M, Buelens A, Vanveggel S, Boven K, et al. Clinical perspective on drug-drug interactions with the non-nucleoside reverse transcriptase inhibitor rilpivirine. AIDS Rev 2013; 15 (2): 87-101.

81.- Imaz A, Podzamczer D. The role of rilpivirine in clinical practice: strengths and weaknesses of the new non nucleoside reverse transcriptase inhibitor for HIV therapy. AIDS Rev 2012; 14 (4): 268-78.
82.- Ripamonti D, Bombana E, Rizzi M. Rilpivirine: drug profile of a second-generation nonnucleoside reverse transcriptase HIV-inhibitor. Expert Rev Anti Infect Ther 2014; 12 (1): 13-29.

83.- Davis J, Langdon G, Layton G, Chong C L, Ndongo M N, Vourvahis M. The effect of lersivirine, a next-generation NNRTI, on the pharmacokinetics of midazolam and oral contraceptives in healthy subjects. Eur J Clin Pharmacol 2012; 68 (11): 1567-72.

84.- Leticee N, Viard J P, Yamgnane A, Karmochkine M, Benachi A. Contraceptive failure of etonogestrel implant in patients treated with antiretrovirals including efavirenz. Contraception 2012; 85 (4): 425-7.

85.- Melvin L. Implanon failure in an HIV-positive woman on antiretroviral therapy resulting in two ectopic pregnancies. Int J STD AIDS 2012; 23 (1): 75 .

86.- Lakhi N, Govind A. Implanon failure in patients on antiretroviral medication: the importance of disclosure. J Fam Plann Reprod Health Care 2010; 36 (3): 181-2.

87.- McCarty E J, Keane H, Quinn K, Quah S. Implanon ${ }^{\circledR}$ failure in an HIV-positive woman on antiretroviral therapy resulting in two ectopic pregnancies. Int J STD AIDS 2011; 22 (7): 413-4.

88.- Landolt N K, Phanuphak N, Ubolyam S, Pinyakorn S, Kerr S, Ahluwalia J, et al. Significant decrease of ethinylestradiol with nevirapine, and of etonogestrel with efavirenz in HIV-positive women. J Acquir Immune Defic Syndr 2014; 66 (2): e50-2.

89.- Grennan T, Walmsley S. Etravirine for HIV-I: addressing the limitations of the nonnucleoside reverse transcriptase inhibitor class. J Int Assoc Physicians AIDS Care (Chic) 2009; 8 (6): 354-63.

90.- Owe-Larsson B, Säll L, Salamon E, Allgulander C. HIV infection and psychiatric illness. Afr J Psychiatry (Johannesbg) 2009; 12 (2): $115-28$

91.- Kakuda T N, Woodfall B, De Marez T, Peeters M, Vandermeulen K, Aharchi F, et al. Pharmacokinetic evaluation of the interaction between etravirine and rifabutin or clarithromycin in HIV-negative, healthy volunteers: results from two Phase 1 studies. J Antimicrob Chemother 2014; 69: 728-34.

92.- Huang L, Parikh S, Rosenthal PJ, Lizak P, Marzan F, Dorsey G, et al. Concomitant efavirenz reduces pharmacokinetic exposure to the antimalarial drug artemether-lumefantrine in healthy volunteers. J Acquir Immune Defic Syndr 2012; 61 (3): 310-6.

93.- Kredo T, Mauff K, Van der Walt JS, Wiesner L, Maartens G, Cohen K, et al. Interaction between artemether-lumefantrine and nevirapine-based antiretroviral therapy in HIV-1-infected patients. Antimicrob Agents Chemother 2011; 55 (12): 5616-23.

94.- Byakika-Kibwika P, Lamorde M, Mayito J, Nabukeera L, Namakula R, et al. Significant pharmacokinetic interactions between artemether/lumefantrine and efavirenz or nevirapine in HIV-infected Ugandan adults. 
J Antimicrob Chemother 2012; 67 (9): 2213-21.

95.- Achan J, Kakuru A, Ikilezi G, Ruel T, Clark T D, Nsanzabana C, et al. Antiretroviral agents and prevention of malaria in HIVinfected Ugandan children. N Engl J Med 2012; 367 (22): 2110-8.

96.- Chijioke-Nwauche I, van Wyk A, Nwauche C, Beshir K B, Kaur H, Sutherland C J. HIV-positive Nigerian adults harbor significantly higher serum lumefantrine levels than HIV-negative individuals seven days after treatment for Plasmodium falciparum infection. Antimicrob Agents Chemother 2013; 57 (9): 4146-50.

97.- Kayentao K, Guirou E A, Doumbo O K, Venkatesan M, Plowe C V, Parsons T L, et al. Preliminary study of quinine pharmacokinetics in pregnant women with malaria-HIV coinfection. Am J Trop Med Hyg 2014; 90 (3): 530-4.

98.- Dooley K E, Park J G, Swindells S, Allen R, Haas D W, Cramer Y, et al; ACTG 5267 Study Team. Safety, tolerability, and pharmacokinetic interactions of the antituberculous agent TMC207 (bedaquiline) with efavirenz in healthy volunteers: AIDS Clinical Trials Group Study A5267. J Acquir Immune Defic Syndr 2012; 59 (5): 455-62.

99.- Svensson E M, Aweeka F, Park J G, Marzan F, Dooley K E, Karlsson MO. Model-based estimates of the effects of efavirenz on bedaquiline pharmacokinetics and suggested dose adjustments for patients coinfected with HIV and tuberculosis. Antimicrob Agents Chemother 2013; 57 (6): 2780-7.

100.-Vourvahis M, Davis J, Wang R, Layton G, Choo H W, Chong C L, et al. Effect of rifampin and rifabutin on the pharmacokinetics of lersivirine and effect of lersivirine on the pharmacokinetics of rifabutin and 25-O-desacetyl-rifabutin in healthy subjects. Antimicrob Agents Chemother 2012; 56 (8): 4303-9.

101.-Curran A, Falcó V, Pahissa A, Ribera E. Management of tuberculosis in HIV-infected patients. AIDS Rev 2012; 14 (4): 231-46.

102.-Michaud V, Ogburn E, Thong N, Aregbe A O, Quigg T C, Flockhart D A, et al. Induction of CYP2C19 and CYP3A activity following repeated administration of efavirenz in healthy volunteers. Clin Pharmacol Ther 2012; 91 (3): 475-82.

103.-Singer E J, Valdes-Sueiras M, Commins D L, Yong W, Carlson M. HIV stroke risk: evidence and implications. Ther Adv Chronic Dis 2013; 4 (2): 61-70

104.-Wenning L A, Hanley W D, Brainard D M, Petry A S, Ghosh K, Jin B, et al. Effect of rifampin, a potent inducer of drug-metabolizing enzymes, on the pharmacokinetics of raltegravir. Antimicrob Agents Chemother 2009; 53(7): 2852-6.

105.-Burger D M. Drug-drug interactions with raltegravir. Eur J Med Res 2009; 14 (Suppl 3): 17-21.

106.-Burger D M. Raltegravir: a review of its pharmacokinetics, pharmacology and clinical studies. Expert Opin Drug Metab Toxicol 2010; 6 (9): 1151-60.

107.-Croxtall J D, Keam S J. Raltegravir: a review of its use in the management of HIV infection in treatment-experienced patients. Drugs 2009; 69 (8): 1059-75.

108.-Mena A, Vázquez P, Castro Á, López S, Bello L, Pedreira J D. Clinical experience of raltegravir-containing regimens in HIV-infected patients during rifampicin-containing treatment of tuberculosis. J Antimicrob Chemother 2011; 66 (4): 951-2.

109.-Lalloo U G. Efavirenz and nevirapine interactions with rifampicin: resolving the dilemmas? Clin Infect Dis 2009; 48 (12): $1760-2$.

110.-Gervasoni C, Riva A, Impagnatiello C, Galli M, Cattaneo D. Is chewed raltegravir an option to care for HIV-infected patients with active tuberculosis? Clin Infect Dis 2013; 57 (3): 480-1.

111.-Pau A K, Boyd S D. Recognition and management of significant drug interactions in HIV patients: challenges in using available data to guide therapy. Clin Pharmacol Ther 2010; 88 (5): 712-9.

112.-Gilliam B L, Riedel D J, Redfield R R. Clinical use of CCR5 inhibitors in HIV and beyond. J Transl Med 2011; 9 Supp11: S9.

113.-Sharma M, Walmsley S L. Raltegravir as antiretroviral therapy in HIV/AIDS. Expert Opin Pharmacother 2014; 15 (3): 395-405.

114.-Funk E K, Shaffer A, Shivakumar B, Sneller M, Polis M A, Masur H, et al. Short communication: Interferon/ribavirin treatment for HCV is associated with the development of hypophosphatemia in HIV/hepatitis C virus-coinfected patients. AIDS Res Hum Retroviruses 2013; 29 (9): 1190-4.

115.- Yeni P, Mills A, Peeters M, Vingerhoets J, Kakuda T N, De Smedt G, et al. Etravirine in the treatment of HIV-1: a clinical overview for healthcare professionals. Curr HIV Res 2010; 8 (7): 564-76.

116.-Song I, Borland J, Min S, Lou Y, Chen S, Patel P, et al. Effects of etravirine alone and with ritonavir-boosted protease inhibitors on the pharmacokinetics of dolutegravir. Antimicrob Agents Chemother 2011; 55 (7): 3517-21.

117.-Rathbun R C, Lockhart S M, Miller M M, Liedtke M D. Dolutegravir, a second-generation integrase inhibitor for the treatment of HIV-1 infection. Ann Pharmacother 2014; 48 (3): 395-403.

118.-Ramanathan S, Abel S, Tweedy S, West S, Hui J, Kearney B P. Pharmacokinetic interaction of ritonavir-boosted elvitegravir and maraviroc. J Acquir Immune Defic Syndr 2010; 53 (2): 209-14.

119.-Cattaneo D, Gervasoni C, Cozzi V, Baldelli S, Fucile S, Meraviglia P, et al. Co-administration of raltegravir reduces daily darunavir exposure in HIV-1 infected patients. Pharmacol Res 2012; 65 (2): 198-203

120.-Serrano López de Las Hazas J I. Drug interactions of new antiretroviral drugs. Farm Hosp 2011; 35 (1): 36-43.
121.-Mateo-Carrasco H, Gálvez-Contreras M C, Fernández-Ginés F D, Nguyen T V. Elevated liver enzymes resulting from an interaction between raltegravir and Panax ginseng: a case report and brief review. Drug Metabol Drug Interact 2012; 27 (3): 171-5.

122.-Havens P L, Stephensen C B, Hazra R, Flynn P M, Wilson C M, Rutledge B, et al; Adolescent Medicine Trials Network (ATN) for HIV/AIDS Interventions 063 study team. Vitamin D3 decreases parathyroid hormone in HIV-infected youth being treated with tenofovir: a randomized, placebo-controlled trial. Clin Infect Dis 2012; 54 (7): 1013-25.

123.-Ibarra-Barrueta O, Palacios-Zabalza I, Mora-Atorrasagasti O, Mayo-Suárez J. Effect of concomitant use of montelukast and efavirenz on neuropsychiatric adverse events. Ann Pharmacother 2014; 48 (1): 145-8.

124.-Aperis G, Paliouras C, Zervos A, Arvanitis A, Alivanis P. Lactic acidosis after concomitant treatment with metformin and tenofovir in a patient with HIV infection. J Ren Care 2011; 37 (1): 25-9.

125.-Morelle J, Labriola L, Lambert M, Cosyns J $\mathrm{P}$, Jouret F, Jadoul M. Tenofovir-related acute kidney injury and proximal tubule dysfunction precipitated by diclofenac: a case of drug-drug interaction. Clin Nephrol 2009; 71 (5): 567-70.

126.-Makinson A, Pujol J L, Le Moing V, Peyriere H, Reynes J. Interactions between cytotoxic chemotherapy and antiretroviral treatment in human immunodeficiency virus-infected patients with lung cancer. J Thorac Oncol 2010; 5 (4): 562-71.

127.-Mallolas Masferrer J, Martínez-Rebollar M, Laguno Centeno M. Treatment of hepatitis C virus in HIV-positive patients. Gastroenterol Hepatol 2011; 34 (8): 558-67.

128.-Pérez V E, Sánchez-Parra C, Serrano Villar S. Etravirine drug interactions. Enferm Infecc Microbiol Clin 2009; 27 (Suppl 2): 27-31.

129.-Dailly E, Allavena C, Bouquié R, Deslandes G, Raffi F, Jolliet P. Effect of efavirenz, nevirapine, etravirine, and raltegravir administration on the pharmacokinetics of ritonavir-boosted darunavir in a population of HIV-infected patients. AIDS Res Hum Retroviruses 2013; 29 (1): 42-6.

130.-Boffito M, Jackson A, Lamorde M, Back D, Watson V, Taylor J, et al. Pharmacokinetics and safety of etravirine administered once or twice daily after 2 weeks treatment with efavirenz in healthy volunteers. J Acquir Immune Defic Syndr 2009; 52 (2): 222-7.

131.-Nina M, Murray J, Gulick R, Filip J, Miller V, Miele P, et al. Novel clinical trial designs for the development of new antiretroviral agents. AIDS 2012; 26 (8): 899-907.

132.-Saag MS. New and investigational antiretroviral drugs for HIV infection: mechanisms of action and early research findings. Top Antivir Med 2012; 20(5): 162-7.

133.-Onakpoya IJ, Heneghan C J, Aronson J K. Delays in the post-marketing withdrawal of drugs to which deaths have been attributed: a systematic investigation and analysis. BMC Med 2015; 13: 26. 